CIA2 and CIA2-LIKE are required for optimal photosynthesis and stress responses in Arabidopsis thaliana

\title{
Gawronski, Piotr
}

2021-02

Gawronski , P , Burdiak , P , Scharff , L B , Mielecki , J , Gorecka , M , Zaborowska , M , Leister , D , Waszczak , C \& Karpinski , S 2021, ' CIA2 and CIA2-LIKE are required for optimal photosynthesis and stress responses in Arabidopsis thaliana ', Plant Journal, vol. 105 , no. 3 , pp. $619-638$. https://doi.org/10.1111/tpj.15058

http://hdl.handle.net/10138/335813

https://doi.org/10.1111/tpj.15058

acceptedVersion

Downloaded from Helda, University of Helsinki institutional repository.

This is an electronic reprint of the original article.

This reprint may differ from the original in pagination and typographic detail.

Please cite the original version. 
1 CIA2 and CIA2-LIKE are required for optimal photosynthesis and stress

\section{responses in Arabidopsis thaliana}

Piotr Gawroński ${ }^{1}$, Paweł Burdiak ${ }^{1}$, Lars B. Scharff ${ }^{2}$, Jakub Mielecki ${ }^{1}$, Magdalena Górecka ${ }^{3}$, Magdalena Zaborowska ${ }^{1}$, Dario Leister ${ }^{4}$, Cezary Waszczak ${ }^{5}$, and Stanisław Karpiński ${ }^{*}$

${ }^{1}$ Department of Plant Genetics, Breeding, and Biotechnology, Warsaw University of Life Sciences, 02-776 Warsaw, Poland

${ }^{2}$ Copenhagen Plant Science Center, Department of Plant and Environmental Sciences, University of Copenhagen, 1871 Frederiksberg C, Denmark

${ }^{3}$ Institute of Biochemistry and Biophysics, Polish Academy of Sciences, Pawińskiego 5a, 02106 Warsaw, Poland

${ }^{4}$ Plant Molecular Biology, Faculty of Biology, Ludwig-Maximilians-University Munich, Großhadernerstraße 2-4, 82152 Planegg-Martinsried, Germany

${ }^{5}$ Organismal and Evolutionary Biology Research Programme, Faculty of Biological and Environmental Sciences, and Viikki Plant Science Centre, University of Helsinki, 00014 Helsinki, Finland

ORCID IDs: 0000-0002-9773-3109 (P.G.); 0000-0003-0252-7116 (P.B.); 0000-00030210-3428 (L.B.S.); 0000-0003-1195-7118 (J.M.); 0000-0001-6585-4929 (M.G.); 0000-00026550-0765 (M.Z.); 0000-0003-1897-8421 (D.L.); 0000-0002-5978-7560 (C.W.); 0000-00024328-1207 (S.K.)

\section{Correspondence to: Stanisław Karpiński: stanislaw_karpinski@sggw.pl}

\section{Summary}

Chloroplast-to-nucleus retrograde signaling is essential for cell function, acclimation to fluctuating environmental conditions, plant growth and development. The vast majority of chloroplast proteins are nuclear-encoded and must be imported into the organelle after synthesis in the cytoplasm. This import is essential for the development of fully functional chloroplasts. On the other hand, functional chloroplasts act as sensors of environmental changes and can trigger acclimatory responses that influence nuclear gene expression. Signaling via mobile transcription factors (TFs) has been recently recognized as a way of communication between organelles and the nucleus. In this study, we performed a targeted reverse genetic screen to identify dual-localized TFs involved in chloroplast retrograde signaling during stress responses. We found that CHLOROPLAST IMPORT APPARATUS 2 (CIA2) has a functional plastid transit peptide and can be located both in chloroplasts 
32 and the nucleus. Further, we found that CIA2, along with its homolog CIA2-like (CIL) are involved

33 in the regulation of Arabidopsis responses to UV-AB, high light, and heat shock. Finally, our results 34 suggest that both CIA2 and CIL are crucial for chloroplast translation. Our results contribute to a 35 deeper understanding of signaling events in the chloroplast-nucleus cross-talk.

\section{Significance}

37 We found that a transcription factor CIA2 can be located in chloroplasts and nucleus and 38 together with its close homolog CIL is involved in protein translation in the chloroplasts and abiotic 39 stress responses.

\section{Keywords}

41 Arabidopsis thaliana, chloroplast retrograde signaling, CIA2, CIL, nonphotochemical 42 quenching, photosynthesis, thermo- and photooxidative stress tolerance, chloroplast translation 


\section{Introduction}

In plants, intracellular communication between the nucleus, chloroplasts, and mitochondria is essential for the regulation and coordination of physiological processes such as growth, development, stress responses, photosynthesis, and respiration (de Souza et al., 2017). Mechanisms that coordinate organellar and nuclear gene expression enable responses to fluctuating or rapidly changing environmental conditions. Besides photosynthesis, chloroplasts play an important role as redox sensors of environmental conditions and trigger acclimatory responses (Li et al., 2009b). Changes in the developmental and metabolic states of chloroplasts or in the redox status of photosynthetic electron carriers can trigger alterations in the nuclear gene expression in a process called retrograde signaling (Chi et al., 2013; Estavillo et al., 2013; Guo et al., 2016). Among the seminal studies that contributed to the discovery of the retrograde signaling was the identification and characterization of barley (Hordeum vulgare) albostrians mutant deficient in HvCMF7 gene (Bradbeer et al., 1979; Hess et al., 1994; Börner 2017; Li et al., 2019). Mutation in the $H v C M F 7$ gene leads to the formation of pigment-deficient plastids and the consequent development of leaf variegation manifested as white stripes along the leaf blade. The green segments of the leaves contain functional chloroplasts, while the white sectors contain ribosome-deficient chloroplasts unable of translation. Analysis of the albostrians mutant demonstrated that the lack of chloroplast ribosomes can influence the expression of nuclear genes encoding for chloroplast proteins, which laid the foundation for the existence of chloroplast-to-nucleus communication (Bradbeer et al., 1979; Hess et al., 1994). Recently, the barley ALBOSTRIANS (HvCMF7) gene was identified by positional cloning and found to encode for a chloroplast-localized transcription factor with a conserved CONSTANS, CO-like, and TOC1 (CCT) domain (Li et al., 2019).

It is now well established that the perturbation of multiple plastid processes, including tetrapyrrole biosynthesis, protein synthesis, reactive oxygen species (ROS) metabolism, and dark and light reactions of photosynthesis, influences the expression of nuclear genes encoding photosynthetic proteins (Pesaresi et al., 2006). Moreover, chloroplast retrograde signaling not only coordinates the expression of nuclear and chloroplast genes, which is essential for chloroplast biogenesis, but also ensures chloroplast vitality in changing environmental conditions (Barajas-López et al., 2013) and triggers the expression of nuclear-encoded genes for other cellular compartments such as the cytoplasm and peroxisomes (Karpiński et al., 1997; Karpiński et al., 1999; Mateo et al., 2004; Mühlenbock et al., 2008; Pogson et al., 2008).

At the mechanistic level, multiple forms of retrograde signaling can be differentiated. Changes in the absorbed light quality and intensity result in rapid changes in the redox state of photosynthetic electron carriers and lead to unbalanced production of ROS such as hydrogen peroxide $\left(\mathrm{H}_{2} \mathrm{O}_{2}\right)$, singlet oxygen, and superoxide anions (Karpiński et al., 1999; Mullineaux et al., 2006; Mühlenbock et al., 
2008; Pogson et al., 2008). Recently, it was proposed that $\mathrm{H}_{2} \mathrm{O}_{2}$ produced in chloroplasts can be directly transported to the nucleus to act as a signaling molecule as its accumulation in both compartments was observed immediately after exposure to light (Caplan et al., 2015; ExpositoRodriguez et al., 2017). Moreover, $\mathrm{H}_{2} \mathrm{O}_{2}$ produced in chloroplasts under drought and excessive light conditions influences the metabolism of 3'-phosphoadenosine 5'-phosphate (PAP), which, upon accumulation, modulates the expression of nuclear stress-responsive genes (Estavillo et al., 2011; Chan et al., 2016). Increased singlet oxygen generation in chloroplasts can also trigger specific retrograde signals. However, due to the high reactivity of singlet oxygen, its half-life is too short to enable direct transport to the nucleus, and it was proposed that carotenoid oxidation product, $\beta$ cyclocitral, acts as a stress signal induced by singlet oxygen produced in grana stacks (Ramel et al., 2012). Moreover, singlet oxygen can be also produced in grana margins where it induces retrograde signaling through two plastid localized proteins, EXECUTER 1 and 2 (Lee et al., 2007; Wang et al., 2016; Dogra et al., 2019).

In addition to signaling via ROS and metabolites, many transcription factors (TFs) were shown to be controlled by signals generated in the organelles. There are two known TFs, ANAC013 and ANAC017, which respond to the mitochondrial redox status (De Clercq et al., 2013; Ng et al., 2013). These TFs are anchored in the endoplasmic reticulum membrane, and in response to signals from mitochondrial complex III, they are released to the nucleus by the proteolytic cleavage of their transmembrane domains. After translocation to the nucleus, ANAC013 and ANAC017 regulate the expression of mitochondrial dysfunction stimulon genes (De Clercq et al., 2013; Ng et al., 2013). Further, it was recently shown that RADICAL-INDUCED CELL DEATH1 (RCD1) interacts with ANAC013 and ANAC017 to integrate ROS signals from chloroplasts and mitochondria (Shapiguzov et al., 2019). Thus, the dual localization of TFs presents a possibility of their function in retrograde signaling.

Early, in silico analyses of Arabidopsis genes encoding putative TFs predicted targeting of at least 48 TFs to the plastids (Wagner and Pfannschmidt, 2006). Later, another in silico-based screen approach predicted that 78 Arabidopsis TFs reside in the plastids (Schwacke et al., 2007). Indeed, several proteins exhibiting dual nuclear-plastid localization might potentially be involved in signal transduction pathways involving regulatory protein storage in the plastids. It was shown that most of the dual-targeted (nucleus and organelle) proteins have functions in the maintenance of DNA, telomere structuring, gene expression, or innate immunity (Krause et al., 2012; Caplan et al., 2015).

109 These in silico studies were supported by in vivo evidence with WHIRLY1 being the first protein to

110 be identified in the nucleus and plastids of the same plant cell (Grabowski et al., 2008); however, its 111 molecular function appears to be compartment-specific. In the nucleus, WHIRLY1 is associated with 112 WRKY53 promoter and acts as a suppressor of leaf senescence (Miao et al., 2013; Huang et al., 2018). 
113 In chloroplasts, WHIRLY1 is present in nucleoid fractions where it might be involved in the 114 maintenance of plastid DNA stability (Maréchal et al., 2009; Krupinska et al., 2014). On the other 115 hand the association of WHIRLY1 with thylakoid membranes, led to formation of a model in which

116 WHIRLY1 was proposed to act as a redox sensor in chloroplast-to-nucleus retrograde signaling 117 (Foyer et al., 2014). Another TF implemented in chloroplast retrograde signaling, the plant 118 homeodomain (PHD) transcription factor PTM (PHD-type TF with transmembrane domains) was 119 shown to accumulate in the nucleus after release from the plastid surface. In the nucleus, PTM was 120 suggested to activate the transcription factor ABA INSENSITIVE 4 (ABI4), thereby providing a way 121 to communicate the plastid status to the nucleus (Sun et al., 2011). However, the roles of PTM and 122 ABI4 in chloroplast-to-nucleus communication were recently questioned (Page et al., 2017; Kacprzak 123 et al., 2019).

124 Chloroplasts contain a few thousand proteins that are involved in photosynthesis, intracellular 125 signaling, and biosynthesis of fatty acids, amino acids, hormones, vitamins, nucleotides, and 126 secondary metabolites (Yu et al., 2008). Most of the chloroplast proteins are encoded by the nuclear 127 genome, synthesized by cytosolic ribosomes, imported into the chloroplast, and targeted to a specific 128 compartment within the chloroplast (Jarvis, 2008). Chloroplast genomes encode about 80 of these 129 proteins, most of which function in photosystems, photosynthetic electron transport, and the 130 organellar gene expression machinery (Scharff and Bock, 2014; Daniell et al., 2016). Although 131 nuclear gene expression involves transcriptional control, it is generally believed that in the course of 132 evolution, the regulation of plastid gene expression has shifted from predominantly transcriptional to 133 predominantly posttranscriptional (Eberhard et al., 2002), although significant transcriptional 134 regulation occurs in chloroplasts (Liere and Börner, 2007). Posttranscriptional control is exerted at 135 the level of mRNA stability and, most importantly, at the level of mRNA translation (Zoschke and 136 Bock, 2018). During chloroplast biogenesis, translational regulation is required for the differentiation 137 of chloroplasts from proplastids during the early development of stem and leaf tissues (Sugiura, 138 2014). At the later stages, in mature chloroplasts, translation is regulated mostly by light and controls 139 chloroplast growth for division in the expanding cells of green tissues. Translational regulation also 140 occurs in response to changing environmental conditions and quite often is essential to repair the 141 photosynthesis machinery (Chotewutmontri and Barkan, 2018). For example, the regulation of $p s b A$ 142 translation encoding for D1 core protein of photosystem II (PSII) is crucial for the repair of 143 photodamaged PSII complexes, whereas the repression of $r b c L$ translation encoding for a large 144 subunit of RuBisCo occurs during oxidative stress (Nickelsen et al., 2014).

145 In this paper, we aimed to characterize dual-localized TFs that can be involved in the 146 communication between the chloroplast and the nucleus. As a result of a targeted reverse genetic 147 screen, we focused on CHLOROPLAST IMPORT APPARATUS 2 (CIA2) and its homolog CIA2- 
148 like (CIL), which encode TFs with conserved CONSTANS, CO-like, and TOC1 (CCT) domain at the

149 C-terminus and a predicted plastid transit peptide at the N-terminus. Our results suggest that CIA2 150 has a dual chloroplast-nuclear localization, and together with CIL plays an important role in the 151 regulation of chloroplast translation, thus influencing photosynthetic electron transport, accumulation 152 of photosynthetic pigments, and stress responses in Arabidopsis thaliana.

\section{Results}

\section{Reverse genetic screen to identify chloroplast-targeted TFs involved in 155 retrograde signaling}

Because chloroplast-to-nucleus signaling pathways are not sufficiently understood, we decided to use the targeted reverse genetic screen to identify retrograde signaling components. To this end, we focused on the identification of dual-localized TFs due to their potential role in chloroplast retrograde signaling. To identify such TFs, we extracted and compared the gene identifiers obtained from three Arabidopsis thaliana plastid proteome databases (Schwacke et al., 2003; Sun et al., 2009b; Myouga et al., 2013) and two transcription factor databases (Pérez-Rodríguez et al., 2009; Jin et al., 2014). This allowed us to identify a set of TFs with potential chloroplast localization. Further, we obtained T-DNA insertion lines for a selected set of genes (Data S1). These mutants were then subjected to conditions promoting chloroplast photooxidative stress, as we hypothesized that mutants lacking efficient communication between the chloroplast and the nucleus (possibly dependent on TFs) would show altered susceptibility to such treatments. During the primary screen, two types of stress treatments were applied: illumination with ultraviolet $\mathrm{AB}$ (UV-AB) and exposure to high light intensity in combination with cold (cHL) (Figure S1). UV-AB significantly impairs photosynthetic electron transport and the general function of photosynthetic machinery (Hollósy, 2002; Caldwell, 1993). At the molecular level, UV-AB damages the ribosomes by crosslinking the cytosolic and chloroplast ribosomal proteins to RNA, thereby transiently inhibiting translation in vivo (Casati and Walbot, 2004; Ferreyra et al., 2010). On the other hand, cHL causes imbalances in photosynthetic reactions resulting in the photoinhibition of photosystem II (PSII) and oxidative stress (Yabuta et al., 2002; Distelbarth et al., 2013). Most of the analyzed mutants were not affected by treatment with $\mathrm{UV}-\mathrm{AB}$ and $\mathrm{cHL}$ as compared to Col-0 (Figure S1); however, based on the phenotypes of mutant lines, we could select eight candidate genes for further investigation (Table S1). Next, the coding sequences of six of the eight candidate genes were cloned, and fusion proteins with C-terminal YFP were transiently expressed under the control of $35 \mathrm{~S}$ promoter in Arabidopsis cotyledons to confirm their putative chloroplast localization (Table S1). Except for p35S::AT5G57180:YFP, which showed chloroplast and nuclear localization (Figure 1a), all of the analyzed fusion proteins were found 
182 APPARATUS2 (CIA2) protein, which was previously found to play a role in protein import into 183 chloroplasts (Sun et al., 2001) and protein synthesis in chloroplasts (Sun et al., 2009a). Importantly, 184 Arabidopsis CIA2 is the closest homologue of barley ALBOSTRIANS (HvCMF7) (Li et al., 2019). 185 The CIA2 protein contains a C-terminal conserved CCT motif, a characteristic feature of one family of TFs involved in light signal transduction (Strayer et al., 2000). The CCT domain contains a putative nuclear localization signal (NLS), which is consistent with the nuclear localization of CIA2 observed earlier (Sun et al., 2001). However, our in silico analysis of CIA2 protein sequence predicted the existence of an $\mathrm{N}$-terminal 59 amino acid-long chloroplast transit peptide, that targets a fraction of the CIA2 into chloroplasts (Figure 1a, Figure 2c and Table S1). The transient expression of full-length CIA2 fused with YFP (p35S::CIA2:YFP) in Arabidopsis seedlings resulted in a relatively weak fluorescence signal observed within chloroplasts (as compared to the one observed in the nuclei, Figure 1a), therefore, we also transiently expressed a 100 amino acid-long N-terminal part of CIA2 fused N-terminally to YFP (p35S::CIA2 ${ }^{1-100}:$ YFP). As a result, we observed a notably higher fluorescence intensity and confirmed the function of CIA2 transit peptide as the presence of the CIA $2^{1-100}:$ YFP fusion protein was detected in chloroplasts (Figure 1b). To confirm our findings in an independent system, we transiently expressed both constructs in tobacco (Nicotiana benthamiana) leaves. In tobacco leaves, the CIA2:YFP localized to chloroplasts and the nucleus (Figure 1c, d), and a similar localization was observed for CIA $2^{1-100}$ :YFP (Figure 1e). We did not manage to detect any fluorescence signal in Arabidopsis plants stably expressing p35S::CIA2:YFP (discussed further), therefore, as an additional confirmation of the functionality of CIA2 cTP we isolated total, stromal and thylakoid protein fractions from Col-0 and plants stably expressing p35S::CIA2 ${ }^{1-100}:$ YFP and separated them using SDS-PAGE followed by Western blot analysis (Figure 1f). Using antibodies against GFP we detected processed CIA2 $2^{1-100}:$ YFP in total and stromal, but not in thylakoid fractions (Figure 1f).

In our reverse genetic screening (Figure 2a,b), line SALK_045340 (hereafter referred to as cia2-4) that harbors T-DNA insertion in the first intron of the CIA2 gene (Figure 2c) showed increased susceptibility to UV-AB as indicated by the lower maximum efficiency of PSII $\left(F_{\mathrm{v}} / F_{\mathrm{m}}\right)$ and higher ion leakage resulting from the induction of cell death (Figure 2a). Similarly, the exposure to cHL reduced the $F_{\mathrm{v}} / F_{\mathrm{m}}$ of cia2-4 to a higher extent than that in the exposure to Col-0 (Figure 2b).

211 Thus, based on the computational prediction of the chloroplast transit peptide (Sun et al., 2009b;

212 Myouga et al., 2013; Schwacke et al., 2003), mutant phenotype observed during the initial screen,

213 and results of the protein localization experiments with the use of YFP-reporter, we decided to focus 214 on CIA2 that can localize in the nucleus and chloroplasts and is required for acclimatory responses 215 to UV-AB and cHL. 


\section{Functional analysis of CIA2 and CIL}

To confirm the role of CIA2 under the analyzed stress conditions, we isolated two additional independent cia2 mutant alleles, cia2-2 (SALK_004037, Col-0 background) and cia2-3 (SGT49, Ler-0 background). In both mutants, the T-DNA was inserted into the first exon of CIA2 (Figure 2c). Because cia2-4 is an intronic allele, we decided to focus our analysis on cia2-2 and cia2-3 alleles. To test whether the incorporation of T-DNA into the CIA2 gene inhibits the accumulation of CIA2 transcript, cia2-2 and cia2-3 mutants were subjected to quantitative RT-PCR analysis using two pairs of primers (Figure 2c,f and Figure S2b,d). Although we did not detect CIA2 transcript in cia2-2 using primers flanking the T-DNA insertion site, we detected a residual amount of PCR product using primers specific to 3'UTR of the CIA2 gene in cia2-2 (Figure 2f). In cia2-3, we did not detect PCR products in both tested primer pairs (Figure S2d). Taken together, these results suggest that full-length CIA2 transcript is not present in either of the analyzed mutants (i.e. cia2-2 and cia2-3).

CIA2 shares 54\% identical amino acids with CIA2-LIKE (CIL, AT4G25990); moreover, CIL also contains C-terminal CCT motif and NLS (Figure 2c) and has a predicted cTP (Figure 2c), albeit the TargetP reliability class for CIL cTP is higher than that for CIA2 (the lower value of reliability class, the safer prediction). To investigate the subcellular localization of CIL we have transiently expressed CIL:YFP under the control of 35S promoter, in Arabidopsis cotyledons and Nicotiana benthamiana leaves and found that in Arabidopsis the majority of the CIL:YFP fusion protein could be found in the nucleolus and nucleoplasm (Figure 3a), while in tobacco the CIL-YFP protein was detected exclusively in nucleolus (Figure 3b). Similarly, no evidence for chloroplast localization was found when $\mathrm{CIL}^{1-100}$ :YFP was transiently expressed in Arabidopsis cotyledons (Figure 3c). Therefore, we conclude that unlike CIA2, which has a dual localization, CIL can be found in the nucleoplasm and nucleolus.

Because of the sequence similarity, CIL was speculated to act redundantly to CIA2 (Sun et al., 2001). To test this hypothesis, we isolated cil-1 (SAIL_228_C01, Col-0 background) and cil-2 (SK14786, Col-4 background) mutants and introduced them into cia2-2 background by crossing. Despite complete lack of full-length $C I L$ transcript in cil-1 and cil-2 mutants (Figure $2 \mathrm{f}$ and Figure S2b), both mutants were phenotypically indistinguishable from their corresponding wild-type (WT) plants. However, the double mutants cia2-2 cil-1 and cia2-2 cil-2 were paler than WT and the corresponding single mutants (Figure $2 \mathrm{~d}$ and Figure S2a). To confirm visual differences, we measured the concentrations of photosynthetic pigments (Sumanta et al., 2014). From this analysis, we deduced that the content of chlorophyll $a$, chlorophyll $b$, and total carotenoids is significantly lower in cia2-2 and cia2-3 (Figure 2e and Figure S2c) mutants, thus supporting previous results obtained for cia2-1 mutant (Sun et al., 2009a). The introduction of cil mutations into cia2-2 background further reduced 
the concentration of photosynthetic pigments (Figure 2d,e and Figure S2a,b), suggesting that CIA2 and CIL synergistically contribute to the accumulation of photosynthetic pigments.

To further confirm that the lack of CIA2 is responsible for the observed phenotype, we introduced constructs that encode CIA2:YFP and YFP:CIA2 fusion proteins under CIA2 native promoter and CIA2:YFP under 35S promoter into cia2-2 background. The complementation lines pCIA2::CIA2:YFP $P_{\text {cia2-2 }}$ and pCIA2::YFP:CIA2 $2_{\text {cia2-2 }}$ exhibited CIA2 transcript level comparable to that of WT plants, whereas a 7-fold increase was observed in p35S::CIA2:YFP plants (Figure 2g). The complementation and overexpression lines were visually indistinguishable from those of WT plants, and spectrophotometric measurement confirmed that the obtained transgenic lines had WTlike levels of photosynthetic pigments (Figure $2 \mathrm{~h}$ ). However, in contrast to results obtained with the use of transient expression systems, we were unable to detect the YFP signal in these complementation lines, which might be related to the high proteolytic turnover rate of CIA2. Taken together, our results strongly suggest that CIA2 and CIL synergistically contribute to accumulation of photosynthetic pigments and thus are required for the proper functioning of photosystems and photosynthesis.

\section{Characterization of cia2 cil responses in chloroplast-targeted stress}

Because cia2-4 mutant was initially selected from the screening using stress conditions that target the photosynthetic apparatus (Figure 2a,b), we set out to test the importance of CIL and CIA2 under stress conditions using chlorophyll fluorescence as a readout. cia2-4 was shown to be more susceptible to UV-AB that induced cell death (determined on the basis of ion leakage and chlorophyll fluorescence measurements) to a significantly higher extent than in Col-0 plants (Figure 2a). In agreement with these data, the exposure of cia2-2 mutant to UV-AB resulted in similar changes in the photosynthetic parameters, which were significantly affected 1 and 2 days after the treatment (Figure 4a-c). Moreover, the introduction of cil mutant into cia2-2 intensified the effect (Figure 4 and Figure S3). Measurements of ion leakage after UV-AB treatment confirmed chlorophyll fluorescence observations suggesting increased cell death in cia2-2 and even stronger effect in cia22 cil-1 and cia2-2 cil-2 (Figure 4 and Figure S3).

To confirm the role of CIA2, and test whether CIL is required for the high light response, we exposed the plants to blue HL (bHL) and monitored $F_{\mathrm{v}} / F_{\mathrm{m}}$ (Figure 5 and Figure S3). At all tested time points, we observed stronger photoinhibition of PSII as evidenced by decreased $F_{\mathrm{v}} / F_{\mathrm{m}}$ in $c i a 2-$ 2 cil-1 and cia2-2 cil-2 (compared to that in WT plants) measured at a level of whole rosette with the strongest effect visible in young (not fully developed) leaves (Figure 5a,b and Figure S3a,b). As increased photoinhibition can result from either increased damage of PSII or decreased PSII repair (Miyata et al., 2015), we monitored recovery after exposure to bHL (Figure 5c). We did not observe 
differences between Col-0 and cia2-2 cil-1 in the rate of $F_{\mathrm{v}} / F_{\mathrm{m}}$ recovery suggesting that increased susceptibility to bHL in cia2-2 cil-1 is not related to the repair of PSII but to increased damage. HLinduced damage of the photosynthetic electron transport chain is often related to the production of ROS such as singlet oxygen, which is mainly produced in PSII antenna, and superoxide anion and $\mathrm{H}_{2} \mathrm{O}_{2}$, which are mainly produced in the vicinity of PSI (Smirnoff and Arnaud, 2019). To simulate conditions of photoinhibition at the PSII side and induce overproduction of ROS, we treated leaf disks of Col-0 and cia2-2 cil-1 with 3-(3,4-dichlorophenyl)-1,1-dimethylurea (DCMU) and methyl viologen (MV) and monitored $F_{\mathrm{v}} / F_{\mathrm{m}}$ (Figure $5 \mathrm{~d}$ ). DCMU noncovalently binds to the quinone B binding side of PSII and inhibits the photosynthetic electron transfer to the plastoquinone (PQ) pool, thus keeping reduced quinone $\mathrm{A}$ and oxidized PQ pool. MV can induce the accumulation of superoxide anion in chloroplast stroma. Upon DCMU and MV treatments, $F_{\mathrm{v}} / F_{\mathrm{m}}$ significantly more decreased in cia2-2 cil-1 than in Col-0, suggesting that the double mutant is more susceptible to photoinhibition and ROS such as singlet oxygen and $\mathrm{H}_{2} \mathrm{O}_{2}$ (Figure 5d). Collectively, our results suggest that CIA2 and CIL are required for an adequate response to conditions that promote photooxidative stress in chloroplasts and trigger cell death signaling.

\section{Role of CIA2 and CIL in the regulation of photosynthesis and NPQ}

Increased susceptibility to HL and UV-AB can be a consequence of impaired nonphotochemical quenching (NPQ), which is responsible for the dissipation of excess light energy as heat (Kulasek et al., 2016; Białasek et al., 2017). Thus, we monitored NPQ using chlorophyll $a$ fluorescence in Col0, cia2-2, cil-1, cil-2, cia2-2 cil-1, and cia2-2 cil-2 (Figure 6 and Figure S4). We observed that NPQ was slightly (but statistically significant) decreased in cia2-2 cil-1 and cia2-2 cil-2 at a level of the whole rosette. Differences were more pronounced when only young, not fully developed leaves were analyzed. In young leaves of cia2-2 cil-1, NPQ was decreased by 40\% when compared to Col-0 (Figure 6a,b). It is well established that the process of NPQ is controlled by a small PSII protein PsbS, which is activated by the acidification of the thylakoid lumen (Niyogi et al., 2005). To check whether this process is impaired in cia2 cil mutants, we measured the proton gradient $(\Delta \mathrm{pH})$ and electric potential $(\Delta \psi)$, which constitute proton motive force (PMF) (Figure 6c-e and Figure S4c-e) at two actinic light intensities (i.e., 160 and $660 \mu \mathrm{mol} \mathrm{m} \mathrm{m}^{-2} \mathrm{~s}^{-1}$ ). Although we did not observe differences in PMF between the analyzed genotypes, we noted that $\Delta \mathrm{pH}$ in cia2-2 as well as in cia2-2 cil-1 and

313 cia2-2 cil-2 was significantly decreased as compared to that in Col-0 (Figure 6e and Figure S4e), 314 which correlates well with decreased NPQ in these genotypes. Because the rosette size of cia2-2 cil-

3151 was slightly smaller than that of Col-0 (Figure 2d, Figure $6 \mathrm{~b}$ ), we also measured $\mathrm{CO}_{2}$ assimilation 316 as a function of light intensity $\left(20-2000 \mu \mathrm{mol} \mathrm{m}^{-2} \mathrm{~s}^{-1}\right)$ and $\mathrm{CO}_{2}$ concentration (20-1500 ppm) (Figure 317 6f,g) and observed moderate but statistically significant decrease in $\mathrm{CO}_{2}$ assimilation in cia2-2 cil-1 
within the tested range of light intensity and $\mathrm{CO}_{2}$ concentration. Taken together, our results strongly suggest that CIA2 and CIL are required for optimal NPQ and proper formation of trans-thylakoid proton gradient as well as for optimal $\mathrm{CO}_{2}$ assimilation.

\section{Chloroplast translation is attenuated in cia2 cil}

It was shown that CIA2 binds to the promoters of genes encoding the chloroplast ribosomal proteins, and decreased expression of these genes was observed in cia2-1 mutant (Sun et al., 2009a). To check whether a similar phenomenon was observed in cia2-2 and if the expression of these genes was dependent on the activity of CIL, we checked the expression of Rps6, Rpl11, Rpl18, and Rpl28 nuclear genes encoding bS6c, uL11c, uL18c, and bL28c chloroplast ribosomal proteins using qRTPCR (Figure 7a). The expression of all analyzed genes was decreased in cia2-2 as compared to that in Col-0 and cil-1. The introduction of cil-1 mutation into cia2-2 led to stronger reduction in the expression of all genes except $R p l 18$, whose transcript was at the same level as that in cia2-2 (Figure 7a). To gain more comprehensive view of gene expression changes, we performed whole transcriptome sequencing (RNA-seq) for Col-0, cia2-2, cil-1, and cia2-2 cil-1 plants. First, we focused on genes encoding for proteins involved in chloroplast translation. Our analysis revealed that the expression of 21 out of 66 chloroplast ribosome genes is inhibited in cia2-2 cil-1 (Figure 7b) including two genes encoded by the chloroplast genome (i.e., rpl2 and rpl32, encoding the ribosomal proteins uL2c and bL32c). Pentatricopeptide repeat (PPR) proteins are involved in every step of chloroplast gene expression, namely transcription, RNA metabolism, and translation (Barkan and Small 2014). Thus, we checked the expression of chloroplast-targeted PPRs in cia2-2 cil-1 and found 13 down-and 7 upregulated PPR genes (Figure 7b). Further, the expression analysis of other genes possibly involved in plastid translation revealed the presence of both up-and downregulated genes (Figure 7b). Taken together, the transcriptome profiling indicates that the lack of CIA2 and CIL leads to altered expression of multiple genes encoding the components of the chloroplast translation machinery.

To further confirm that CIA2 and CIL are required for optimal chloroplast translation, we subjected Col-0 and cia2-2 cil-1 to polysome loading assays. We examined the ribosomal loading of $p s b D$ mRNA (encoding the D2 protein of PSII) using sucrose gradient fractionation followed by northern blot analysis (Figure 7c). This analysis showed that $p s b D$ mRNA was underrepresented in the polysomal fractions in cia2-2 cil-1 compared to that in Col-0, and the mRNA was shifted toward lighter (monosome) fractions, suggesting that $p s b D$ mRNA was associated with fewer ribosomes and that its translation was reduced in cia2-2 cil-1. Furthermore, as multiple lines of evidence indicate that CIA2 and CIL are involved in chloroplast translation, we also tested the growth of Col-0, cia22, cil-1, and cia2-2 cil-1 in media supplemented with spectinomycin, which inhibits this process. 
352 Spectinomycin binds the 30S subunit of the 70S ribosome and prevents translocation of peptidyl-

353 tRNA from A to P site; consequently, many Arabidopsis translation mutants showed increased 354 susceptibility to this antibiotic (Parker et al., 2014). In agreement with the results of ribosome loading 355 experiments, we observed severe inhibition of pigment accumulation in cia2-2 cil-1 grown in media 356 supplemented with spectinomycin (Figure 7d), which suggests that CIA2 and CIL play a role in 357 plastid translation.

358 The decreased chlorophyll content in young leaves and inhibited translation in cia2-2 cil-1 led 359 us to analyze chloroplast ribosomal RNA (rRNA) maturation. In Arabidopsis chloroplasts, all rRNAs 360 are encoded by the polycistronic $r r n$ operon (Bollenbach et al., 2007). Upon processing of the initial 361 transcript by distinct endo-and exonucleases, tRNAs, precursors of $16 \mathrm{~S}$ and 5S rRNAs and bicistronic 362 23S-4.5S intermediate are created (Figure 8a). The $23 \mathrm{~S}-4.5 \mathrm{~S}$ precursor is cut to produce $4.5 \mathrm{~S}$ and $23 \mathrm{~S}$ 363 rRNA fragments. The maturation of 23S rRNA is followed by the introduction of two gaps ("hidden 364 breaks") producing three distinct parts of $0.4,1.1$, and $1.3 \mathrm{~kb}$; however, the functional relevance of 365 this postmaturation processing is not clear (Bollenbach et al., 2007; Fristedt et al., 2014). The 366 capillary electrophoresis of RNA isolated from young leaves showed that the 23S rRNA 1.1 and 1.3 $367 \mathrm{~kb}$ species of and the 16S rRNA accumulate to lower levels in cia2-2 and cia2-2 cil-1 mutants as 368 compared to that in WT plants (Figure 8b). These results suggest that the expression and/or processing 369 of $16 \mathrm{~S}$ and 23S rRNAs are dependent on CIA2 and CIL. To further characterize rRNA maturation, 370 we performed northern blot analysis with probes specific to $16 \mathrm{~S}$ and $23 \mathrm{~S}$ rRNAs (Figure $8 \mathrm{c}$ ). We 371 observed only slightly lower level of 16S rRNA in cia2-2 cil-1 without accumulation of its $1.7 \mathrm{~kb}$ 372 precursor suggesting that the processing of the 16S rRNA is not impaired (Figure 8c). In agreement 373 with the results of capillary electrophoresis, we observed decreased accumulation of $0.4,1.1,1.3$, and $3741.7 \mathrm{~kb}$ species and increased accumulation of $2.4 \mathrm{~kb}$ fragment of $23 \mathrm{~S}$ rRNA in cia2-2 cil-1 (Figure $3758 \mathrm{c}$ ). These results suggest that gap incorporation between $1.1 \mathrm{~kb}$ and $1.3 \mathrm{~kb}$ fragments is less efficient 376 in cia2-2 cil-1 mutant. To confirm these results, we performed qRT-PCR analysis with primers spanning hidden breaks (Figure 8d) and obtained confirmatory results.

378 Next, we tested whether the perturbations of chloroplast translation are linked to the decreased 379 NPQ observed in cia2 cil (Figures 6a and S5). For this, we measured NPQ in well-characterized 380 chloroplast translation mutants prpl11-1, prps1-1, psrp5-R1, and rps17-1 (Pesaresi et al., 2001; 381 Romani et al., 2012; Tiller et al., 2012; Tadini et al., 2016) (Figure S5). However, in contrast to cia2 $382 \mathrm{cil}, \mathrm{NPQ}$ in these mutants was increased as compared to that in Col-0 plants (Figure S5). These results 383 suggest that the observed decrease in NPQ in cia2 cil mutant is not related to inhibited chloroplast 384 translation. Taken together, our results suggest that the translation of chloroplast mRNA and the 385 maturation and accumulation of $23 \mathrm{~S}$ rRNA are influenced by CIA2 and CIL. 
To our surprise, RNA-seq analysis showed that many genes induced in cia2-2 cil-1 were annotated as heat shock proteins (HSPs) (Data S2). Further, the gene ontology (GO) enrichment analysis demonstrated that GO terms related to heat response and acclimation were overrepresented among genes induced in cia2-2 cil-1 (Figure 9a). To confirm the RNA-seq results, we performed qRT-PCR analysis to assess the transcript level of HEAT SHOCK TRANSCRIPTION FACTOR A2 (HSFA2) and HEAT SHOCK PROTEIN 70-4 (HSP70-4) in cia2-2, cia2-3, cil-1, cia2-2 cil-1, and corresponding WT plants (Figure 9b). Our results confirmed strong induction of both genes in cia2 mutants, and even stronger induction was observed in cia2-2 cil-1 double mutant (Figure 9b). To check whether increased expression of many HSPs can confer thermotolerance, we grew cia2-2, cil1, cia2-2 cil-1, and Col-0 seedlings on Petri dishes and exposed them to heat stress $\left(45^{\circ} \mathrm{C}\right)$ for 20 $40 \mathrm{~min}$ (Figure 9c,d). Exposure to $45^{\circ} \mathrm{C}$ for $30 \mathrm{~min}$ resulted in almost complete bleaching and growth inhibition of Col-0 plants (Figure 9c). A similar phenotype was observed in the case of cia2-2 and cil-1 mutants; however, cia2-2 cil-1 plants were affected to a significantly lower extent, and nearly all individuals retained cotyledon and leaf pigmentation (Figure 9c,d). Furthermore, at other tested time points, cia2-2 cil-1 was more resistant to heat stress than the remaining genotypes (Figure 9c,d). These results suggest that CIA2 and CIL synergistically contribute to the modulation of thermotolerance presumably through the regulation of HSP expression.

\section{Discussion}

In the present study we have utilized a reverse genetic approach to identify regulators of chloroplast retrograde signaling. With a similar aim, Ruckle et al., (2012) utilized the transcriptome profiling data to select the candidate genes, that were later subjected to phenotypical analysis to identify genes potentially involved in plastid-to-nucleus communication. Our selection strategy was specifically targeted at identification of dual-targeted transcription factors, therefore, we focused on computational prediction of chloroplast transit peptides in known transcription factors. The initial selection was followed by screening of a collection of T-DNA mutant lines for a selected set of transcription factors (Data S1) under conditions promoting chloroplast stress (Figure S1).

As a result of our screening strategy we focused on CIA2 and found that it has a functional cTP which, in transient expression experiments, targets a fraction of CIA2:YFP fusion protein to chloroplasts of Arabidopsis seedlings and tobacco leaves (Figure 1c, Figure 2c). In agreement with our observation, recently characterized barley CIA2 homolog, $\mathrm{HvCMF}$, was also shown to be localized in chloroplasts (Li et al., 2019). In the first attempt to determine the subcellular localization 418 of CIA2, the GUS:CIA2 fusion protein was found to reside exclusively in the nucleus (Sun et al., 419 2001). We speculate that the discrepancy between our data and the previous work might be related to 
420 the masking of CIA2 cTP by the GUS fused to CIA2 N-terminus, thus inhibiting the import of 421 GUS:CIA2 fusion protein into chloroplasts (Sun et al., 2001). From the results of our 422 complementation test, we were unable to determine the significance of CIA2 chloroplast localization 423 because the expression of both constructs, pCIA2::CIA2:YFP and pCIA2::YFP:CIA2, restored the 424 accumulation of photosynthetic pigments in cia2-2 mutant (Figure 2h). Thus, we speculate that at 425 least with regard to the determination of the levels of photosynthetic pigments, the chloroplast 426 localization of CIA2 is not crucial. Interestingly, despite clear complementation effect and restoration 427 of CIA2 transcript level, we did not observe YFP signal in stable transformants expressing CIA2:YFP 428 fusion either under native or 35S promoter, suggesting that the CIA2 protein level might be subject 429 to strict posttranslational control. Such stringent control of protein level was documented earlier for 430 Golden 2-like (GLK) transcription factors that are involved in expression of nuclear-encoded 431 chloroplast-localized proteins and photosynthesis-related genes in Zea mays, Physcomitrella patens, 432 and Arabidopsis thaliana (Yasumura et al., 2005; Waters et al., 2009). According to the proposed 433 model, damaged chloroplasts, send an unknown signal which activates the ubiquitin-proteasome 434 system leading to GLK1 degradation, thereby optimizing the import of photosynthesis-related 435 proteins into chloroplasts (Tokumaru et al., 2017). A similar scenario i.e. the inability to detect the 436 fluorophore-tagged proteins despite complementation of the mutant phenotype, was observed also for 437 ABI4 (Finkelstein et al., 2011) and RCD1 (Jaspers et al., 2009), suggesting that certain regulatory 438 proteins might be unstable and/or degraded by the proteasome, thus preventing their detection under 439 standard conditions. A comparable phenomenon was observed in the case of another key player of 440 retrograde signaling, GUN1, which accumulates in chloroplasts only at a very early stage of leaf 441 development (Wu et al., 2018). Interestingly, unlike CIA2:YFP, CIL:YFP was observed in nucleolus 442 and nucleoplasm (Figure 3) suggesting that CIA2 and CIL have, at least to some extent, distinct 443 molecular roles in the cell. However, both proteins can be localized in the nucleoplasm suggesting 444 possible involvement in the same cellular process, which could explain observed synergistic effects 445 in the cia2 cil double mutant.

446 Our data show that CIA2 and its close homolog CIL play a relevant function in the chloroplast 447 biogenesis process (Figure 2). The cia2 cil double mutant exhibited a pale green phenotype, which 448 was much more pronounced than that observed in single cia2 mutants, suggesting that both of these 449 proteins contribute to chloroplast biogenesis. Double mutant plants displayed a significantly lower 450 concentration of chlorophyll $a$, chlorophyll $b$, and carotenoids (Figure 2 and Figure S2), suggesting 451 impairment in the conversion of light into chemical energy. A similar phenotype was observed in 452 plants lacking the activity of GLK transcription factors. Like cia2 cil, the glk1 glk2 double mutants 453 were pale green and deficient in the formation of the photosynthetic apparatus (Waters et al., 2009). 454 Our RNA-seq and qRT-PCR-based expression data revealed a significant transcriptional 
455 downregulation of chloroplast ribosome genes in cia2 cil plants. Almost one-third of 66 chloroplast 456 ribosome protein genes were significantly repressed in cia2-2 cil-1. These data are consistent with 457 previous reports showing that CIA2 binds to the promoters of genes encoding chloroplast ribosomal 458 proteins (Sun et al., 2009a) and support a positive regulatory role of CIA2 in chloroplast translation. 459 Chloroplast ribosomal proteins are encoded by both nuclear and chloroplast genomes (Zoschke and 460 Bock, 2018). Interestingly, the majority of ribosomal genes downregulated in cia2 cil are nuclearencoded. This, together with partial chloroplast localization of CIA2 (Figure 1), suggests that CIA2 might act as chloroplast sensor of the environmental stimuli and mediates chloroplast-dependent adaptive responses (Estavillo et al., 2013). On the other hand, the phenotypic similarities between cia2 cil, glk1 glk2 double mutant and albostrians appear to be related to the inhibition of chloroplast translation which suggests that in addition to operational signaling in response to environmental perturbations (i.e. HL and UV-AB) CIA2 might be involved in biogenic signaling. Similarly, whirlyl mutants show several chloroplast phenotypes including the lack of chloroplast ribosomes and reduced compactness of nucleoids in chloroplasts (Prikryl et al., 2008; Krupinska et al., 2014). The WHIRLY1 was also suggested to be involved in chloroplast to nucleus retrograde signalling (Foyer et al., 2014). Future research to determine the functional significance of the chloroplast localization of CIA2, and its potential link with established retrograde signalling pathways is required to precisely determine the function of CIA2.

More than $95 \%$ of the chloroplast proteins are nuclear-encoded (Jarvis and Robinson, 2004) and transported across the double chloroplast membrane due to the activity of a specialized translocon complex (Nakai, 2015; Paila et al., 2015). This mechanism is essential for chloroplast biogenesis and requires coordinated action of multiple proteins. Previous reports revealed a positive regulatory effect of CIA2 on the transcription of the translocon genes Toc33 and Toc75 in leaves (Sun et al., 2001; Sun et al., 2009a), suggesting the role of CIA2 in protein import into the chloroplast. Inefficient chloroplast protein import causes cytosolic overaccumulation of preproteins, which results in the activation of chaperones such as HSP70 and HSP90, which were recently postulated to be key components of the chloroplast retrograde signaling pathway (Wu et al., 2019). Accordingly, we observed strong transcriptional induction of cytosolic chaperones and increased thermotolerance in cia2 cil mutant (Figure 9). On the other hand, it was demonstrated that thermotolerance is regulated by chloroplast signals that depend on the redox state of the PQ pool or hydrogen peroxide produced in chloroplasts (Dickinson et al., 2018). The redox state of the PQ pool and $\mathrm{H}_{2} \mathrm{O}_{2}$ are also involved in high-light acclimatory responses (Karpiński et al., 1999; Mühlenbock et al., 2008; Gilroy et al., 2016). Experimentally, it is almost impossible to separate foliar heat shock from high-light responses, because exposure to high light for a few seconds significantly warms up Arabidopsis leaves due to the dissipation of energy as heat (Kulasek et al., 2016). Therefore, it is interesting that CIA2 and CIL 
antagonistically influence high-light and UV-B acclimation versus thermotolerance in ambient light.

Abolished expression of $C I A 2$ and $C I L$ genes in double mutant causes growth reduction, impaired acclimation to high light and UV-AB stresses, and photosynthesis dysfunction, while it can cause thermotolerance expressed as seedling survival rate at high temperatures. However, we were not able

494 to identify the precise role of CIA2 and CIL in the antagonistic regulation of these processes, and we 495 plan to perform field experiments similar to those we did for cell death conditional regulators (i.e. 496 LSD1, EDS1, PAD4) (Wituszyńska et al., 2013). These proteins, depending on growing conditions, 497 differently regulate chloroplast retrograde cell death signaling for growth, photosynthesis, high-light 498 and UV-B acclimation, water use efficiency, and seed yield (Wituszyńska et al., 2013; Wituszyńska et al., 2015; Bernacki et al., 2019).

Finally, our data indicate that CIA2 and CIL influence chloroplast translation by the regulation of ribosome assembly and maturation as cia2 cil double mutants displayed a disturbed accumulation of 23S rRNA (Figure 8). In plant chloroplasts, 23S rRNA constitutes a component of a large (50S) subunit of chloroplast ribosomes. The rRNA undergoes postmaturation processing, which includes a site-specific cleavage that generates gapped, discontinuous rRNA molecules (Nishimura et al., 2010). The maturation process is followed by the removal of a specific region and introduction of a gap (the so-called hidden break) into the $23 \mathrm{~S}$ rRNA (Bollenbach et al., 2005). This molecule is split into four major fragments of 1.7, 1.3, 1.1, and $0.4 \mathrm{~kb}$. Chloroplasts of all dicotyledonous plants have hidden breaks at similar locations. Several proteins have been reported to bind to a $23 \mathrm{~S}$ rRNA segment close to the hidden break sites (Bieri et al., 2017). We observed an increased accumulation of the $2.4 \mathrm{~kb}$ $23 \mathrm{~S}$ rRNA species and a reduced occurrence of the hidden break in this species (Figure 8c,d). Interestingly, the expression of the two genes Rpl19.1 and Rpl19.2 (Figure 6b), which encode the ribosomal protein bL19c binding close to the hidden break (Bieri et al., 2017), was reduced. This indicates that the lack of bL19c could influence the rRNA structure in a way that makes it less susceptible to the hidden break incorporation. The lack of bL19c could be one of the causes of the impaired biogenesis of the $50 \mathrm{~S}$ subunit as shown by the reduced accumulation of the $23 \mathrm{~S}$ rRNA (Figure 8c,d). The deficiencies in rRNA maturation observed in cia2-2 cil-1 double mutant suggest that CIA and CIL might play a role in this process, either directly by binding and processing $23 \mathrm{~S}$ rRNA precursor or more likely indirectly by regulating the expression of ribosomal proteins.

In conclusion, the presented results demonstrate high complexity and elegancy of chloroplast retrograde signaling and anterograde nuclear responses that depend on TFs such as CIA2 and CIL.

521 This complexity is not only observed on the molecular and biochemical levels but also on the 522 physiological level of acclimatory responses as well as on the regulation of chloroplast biogenesis 523 and plant growth and development. To better understand the interdependence of these regulatory 524 processes, further interdisciplinary studies on the function of these proteins are needed. 


\section{Experimental procedures}

\section{Plant material and growth conditions}

Arabidopsis thaliana ecotypes Columbia-0 (Col-0), Columbia-4 (Col-4), and Landsberg erecta 528 (Ler-0) were used as controls for analyzed mutants. cia2-2 (SALK_004037), cia2-3 (SGT49), cia24 (SALK_045340), cil-1 (SAIL_228_C01), and cil-2 (SK14786) were ordered from the Nottingham Arabidopsis Stock Centre (NASC) and genotyped using primers shown in Table S2. The cia2-2 cil1 and cia2-2 cil-2 double mutants were generated by crossing. Plants were grown on Jiffy pots (Jiffy

Products) for 3-4 weeks in a growing chamber in long-day photoperiod (16 h/8 h) under constant white light of $140-160 \mu \mathrm{mol}$ photons $\mathrm{m}^{-2} \mathrm{~s}^{-1}$ at $22{ }^{\circ} \mathrm{C}$.

\section{4}

\section{Databases}

In this work, we used information extracted from proteome databases to identify chloroplast proteins [Plant Proteomics Database at Cornell (http://ppdb.tc.cornell.edu/) (Sun et al., 2009b); The Chloroplast Function Database II (http://rarge-v2.psc.riken.jp/chloroplast/) (Myouga et al., 2013), and ARAMEMNON (http://aramemnon.uni-koeln.de) (Schwacke et al., 2003)] and TF databases to look for annotated $\mathrm{TFs}$ in Arabidopsis [The Plant Transcription Factor Database (http://plntfdb.bio.uni-potsdam.de) (Pérez-Rodríguez et al., 2009) and PlantTFDB (http://planttfdb.cbi.pku.edu.cn/) (Jin et al., 2014)].

\section{Vector constructions and Arabidopsis transformation}

CIA2 and CIL full CDSs were amplified with primers listed in Table S2 by PCR using cDNA from Col-0 plants. PCR products of expected length were purified from agarose gel and inserted into the entry clone using the pENTR/D-TOPO cloning kit (Invitrogen). DNA fragments encoding for Nterminal 100 aa as well as predicted cTP were amplified from plasmids carrying CIA2 and CIL CDSs. Next, they were subcloned into pGWB641 vector (Nakagawa et al., 2007) using Gateway® LR Clonase $^{\mathrm{TM}}$ II enzyme mix (Invitrogen). These constructs were introduced into Agrobacterium fabrum (GV3101). The vector was transformed into Col-0 and/or cia2-2 mutant by the floral dip method. The T3 generation of homozygous transgenic plants was obtained and used for further experiments. The complementation lines were obtained in a similar manner, but in the final step, the promoter, CDS and YFP sequences were cloned into the pK7m34GW binary vector and transformed into Agrobacterium.

In parallel, whole CIA2 and the CIA2 fragment encoding for the 100 aa $\mathrm{N}$-terminal part of CIA2 were amplified from cDNA using USER compatible (Nour-Eldin et al., 2006) primers and the 
improved Pfu X7 polymerase (Nørholm, 2010). PCR fragments were cloned into the pLIFE001 vector (Silvestro et al., 2013) and introduced into Agrobacterium (GV3101) for generation of stably transformed line expressing p35S::CIA ${ }^{1-100}:$ YFP construct.

\title{
Chlorophyll a fluorescence, $\mathrm{CO}_{2}$ assimilation, and $\Delta \mathrm{pH}$ measurements
}

\author{
Chlorophyll $a$ fluorescence parameters were measured in dark-acclimated (30 min) plants using
} PAM FluorCam 800 MF PSI device (Brno, Czech Republic) as described earlier (Gawronski et al., 2014). $\mathrm{CO}_{2}$ assimilation was measured as described earlier (Burdiak et al., 2015). The electrochromic pigment shift (ECS) was measured using DUAL-PAM 100 equipped with P515/535 module (Walz), which allows the simultaneous measurement of the dual beam 550-515 nm signal difference (Schreiber and Klughammer, 2008). Before measurement, the plants were dark acclimated for $30 \mathrm{~min}$ and subsequently illuminated with red actinic light of $160 \mu \mathrm{mol}$ photons $\mathrm{m}^{-2} \mathrm{~s}^{-1}$ for $20 \mathrm{~min}$. At the beginning of each measurement, the actinic light was turned off for $23 \mathrm{~s}$, and three single turnover (ST) flashes were applied. Next, the red actinic light $\left(160 \mu \mathrm{mol}\right.$ photons $\left.\mathrm{m}^{-2} \mathrm{~s}^{-1}\right)$ was turned on, and after $4 \mathrm{~min}$, it was again turned off to determine total ECS, $\Delta \mathrm{pH}$, and $\Delta \Psi$ as described previously (Herdean et al., 2016). Immediately after the first measurement, the ECS signal was recorded using the same protocol but at a higher actinic light intensity $\left(660 \mu \mathrm{mol}\right.$ photons $\left.\mathrm{m}^{-2} \mathrm{~s}^{-1}\right)$. The ECS signal was normalized to the highest ST peak recorded at the beginning of each measurement.

\section{Photosynthetic pigment analysis}

For pigment analysis, approximately 15-20 mg of tissue was collected from the sixth leaf of 34-week-old plants. Tissue was immediately frozen in liquid nitrogen and ground in the presence of methanol. Absorbance was measured using Multiskan GO (Thermo Fisher Scientific) spectrophotometer after the clarification of the supernatant by centrifugation. Photosynthetic pigment concentrations were calculated as reported previously (Sumanta et al., 2014).

\section{Stress treatments and ion leakage}

High-light stress was applied to whole plants using blue light (455 nm) of $1100 \mu \mathrm{mol}$ photons $\mathrm{m}^{-2} \mathrm{~s}^{-1}$ for the indicated time. As a light source, SL3500-B-D LED array was used (PSI, Brno, Czech Republic). After illumination, the plants were dark acclimated for $20 \mathrm{~min}$, and chlorophyll a fluorescence was measured as described above. For recovery experiments, we used blue light of 2500 photons $\mathrm{m}^{-2} \mathrm{~s}^{-1}$ for $2.5 \mathrm{~h}$. After this treatment, the plants were placed in the growing chamber for an indicated period of time followed by $20 \mathrm{~min}$ dark acclimation and chlorophyll $a$ fluorescence measurement. For UV-AB stress, UVC 500 Crosslinker (Hoefer Pharmacia Biotech, USA) equipped with UV-A (TL8WBLB, Philips) and UV-B lamps (G8T5E, Sankyo Denki) was employed. The 


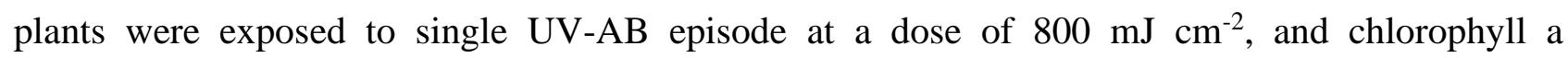

589 fluorescence was analyzed 24 and $48 \mathrm{~h}$ after stress. Ion leakage was also used to analyze cell death

$59048 \mathrm{~h}$ after UV-AB stress as described before (Burdiak et al., 2015) with the following modification:

591 total ion leakage was evaluated after freezing at $-80{ }^{\circ} \mathrm{C}$ overnight.

\section{Herbicide and antibiotic treatments}

593 Leaf disks cut from 4-to 5-week-old plants were treated with $62.5 \mu \mathrm{M}$ of 3-(3,4

594 dichlorophenyl)-1,1-dimethylurea (DCMU) and $5 \mu \mathrm{M}$ of N,N'-dimethyl-4,4'-bipyridinium dichloride

595 (methyl viologen, MV) with 0.05\% Tween-20 and were kept in the growing chamber under ambient

596 light between measurements. The maximum efficiency of PSII $\left(F_{\mathrm{v}}{ }^{\prime} / F_{\mathrm{m}}{ }^{\prime}\right)$ of leaf disks was measured

597 using FluorCam 800MF (Photon Systems Instruments, Czech Republic). As a control, we used leaf

598 disks incubated in identical conditions without herbicides.

599 For spectinomycin-resistance experiments, seeds were surface sterilized in $50 \%(\mathrm{v} / \mathrm{v})$ 600 commercial bleach, washed five times with sterile water and stratified for $2-4$ days at $4{ }^{\circ} \mathrm{C}$ in $0.1 \%$ 601 (w/v) agarose solution. Next, seeds were sowed onto 1/2 MS media supplemented with $1 \%$ sucrose 602 and $1.25 \mathrm{mg} / \mathrm{L}$ spectinomycin (Duchefa, S0188.0025) and grown for two weeks in standard 603 conditions.

\section{RNA isolation and transcriptome profiling}

For RNA isolation, only young (not fully developed) leaves were used. In the middle of the photoperiod, the leaves were detached and frozen in liquid nitrogen. For HL treatment, whole rosettes were illuminated with blue light (length $1100 \mu \mathrm{mol}$ photons $\mathrm{m}^{-2} \mathrm{~s}^{-1}$ ) for $1 \mathrm{~h}$. Immediately following the treatment, young leaves were detached and frozen in liquid nitrogen. Leaf samples represent three biological replicates, and each contained leaves from at least three individual plants. Frozen leaves were ground using mortar and pestle in liquid nitrogen, and RNA was isolated using Spectrum ${ }^{\mathrm{TM}}$

611 Plant Total RNA Kit (Sigma). The quality and quantity of total RNA were evaluated using 612 Experion $^{\mathrm{TM}}$ StdSens RNA Kit (Bio-Rad), and the samples with RQI value higher than 9.0 were used 613 for RNA-seq library construction using TruSeq RNA Sample Prep Kit v2 (Illumina). RNA-seq 614 libraries were sequenced in 100 bp paired-end reads using Illumina HiSeq 4000. RNA-seq library 615 preparation and sequencing were conducted by Macrogen (Seoul, Korea). Reads were pseudomapped 616 to Arabidopsis thaliana cDNAs (Ensembl, TAIR 10, release 35) using Salmon software (Patro et al., 617 2017). Transcript-level abundances were imported into R using tximport (Soneson et al., 2016) and 618 analyzed using DESeq2 package (Love et al., 2014). Significantly enriched GO terms were identified 619 in up-/downregulated gene sets using PANTHER Classification System at http://geneontology.org/. 
RNA-seq data are provided at (uploaded upon manuscript acceptance).

\section{2}

\section{Quantitative real-time PCR}

$$
\text { RNA for qRT-PCR was isolated as described above. RNA was treated with DNase (TURBO }
$$
DNA-free ${ }^{\mathrm{TM}}$ Kit, Thermo Fisher Scientific) to remove residual DNA. Next, cDNA was synthesized from $1 \mu \mathrm{g}$ of total RNA using High-Capacity cDNA Reverse Transcription Kit (Applied Biosystems). PCRs were run on a 7500 Fast Real-Time PCR System (Applied Biosystems) using PerfeCTa ${ }^{\mathrm{TM}}$ SYBR ${ }^{\circledR}$ Green FastMix ${ }^{\mathrm{TM}}$, Low $\mathrm{ROX}^{\mathrm{TM}}$ (Quanta BioSciences) according to the manufacturer's instructions. All primers used in qRT-PCR are listed in Table S2. The amplification efficiency of primers was calculated on the basis of the amplification curve using LinRegPCR software (Ramakers et al., 2003). Relative expression was calculated using EasyqpcR package (http://www.bioconductor.org/packages/release/bioc/html/EasyqpcR.html) with PP2AA3 and TIP41L as reference genes.

\section{Northern blot and polysome analysis}

Northern blot analysis and polysome analysis were done as described previously (Fristedt et al., 2014) but using Hybond-N membranes (GE Healthcare Life Sciences). Probes were amplified from total plant DNA using gene-specific primers (Table S2), radioactively labeled using the Megaprime DNA Labeling System (GE Healthcare Life Sciences), and hybridized at $65{ }^{\circ} \mathrm{C}$.

\section{Protein fractionation and Western blot}

Chloroplasts were isolated from Col-0 and stable p35S::CIA ${ }^{1-100}:$ YFP Arabidopsis thaliana plants by differential centrifugation on 40\% Percoll layer following published protocol (Klinkenberg 2014, Klinkenberg et al., 2014). Briefly, total protein extracts were obtained by grinding $100 \mathrm{mg}$ of plant tissue in $400 \mu \mathrm{l}$ of PBS. Protein concentration in samples were quantified using Bradford Assay (Sigma-Aldrich). Samples were mixed with 4x Laemmli Buffer (Bio-Rad) and denaturated in $95^{\circ} \mathrm{C}$ for 10 mins prior loading onto gel. Samples were separated by SDS-PAGE on $10 \%$ polyacrylamide gel, each well was loaded with $20 \mu \mathrm{g}$ of proteins. After electrophoresis samples were transferred from gel to Immobilon P PVDF membrane (Merck) by semi-dry transfer. Next, 1:10 000 Dilution of primary anti-GFP (G1544, Sigma-Aldrich), anti-RbcL (AS03 037, Agrisera), anti-D1 (AS05 084, Agrisera) and secondary anti-rabbit antibodies (Thermo Fisher Scientific) were used. Signal from chemiluminescence was detected using Pierce ECL Plus Western Blotting Substrate (Thermo Fisher Scientific) and ChemiDoc Imaging Systems (Bio-Rad). To confirm that wells were loaded with same 
amount of protein analogical gel were stained with QC Colloidal Coomassie Stain (Bio-Rad) and PVDF membrane after transfer were stained with Ponceu S (Sigma-AldrichS) before blocking.

\section{3}

654

655

656

657

658

659

660

661

662

663

\section{4}

665

666

667

668

669

670

671

672

673

674

675

\title{
Transient expression in Arabidopsis seedings and tobacco leaves
}

\author{
For transient expression CIA:YFP, CIA ${ }^{1-100}:$ YFP, CIL:YFP or $\mathrm{CIL}^{1-100}:$ YFP fusion proteins
} in Arabidopsis, 4-days-old Arabidopsis seedlings were infiltrated using Agrobacterium (GV3101) strain carrying appropriate constructs using the FAST method (Li et al., 2009a).

For expression in tobacco leaves 4- to 5-week old plants were infiltrated with Agrobacterium strain GV3101 carrying appropriate constructs. Suspensions for infiltration were prepared in infiltration buffer (10 mM MgCl $2,10 \mathrm{mM}$ MES (pH 6.5), $100 \mu \mathrm{M}$ acetosyringone) from overnight cultures supplemented with appropriate antibiotics and consisted of mixture of 19K strain for RNA silencing suppression (Te et al., 2005) at the final OD600 $=0.2$ and analysed strain with CIA:YFP, CIA $^{1-100}:$ YFP, CIL:YFP or CIL ${ }^{1-100}:$ YFP overexpression at the final OD600 $=0.8$. After infiltration plants were kept in low light.

\section{Confocal microscopy}

Sections from central regions next to the mid-vein of fully expanded tobacco leaves were taken for analyses 3 days after agro-infiltration. Observations were carried out using confocal laser scanning inverted microscope Nikon TE2000E EZ-C1 equipped with 60x and 100x Plan-Apochromat oilimmersion objectives. Fluorescence was excited with the 488-nm argon ion laser. Signals were detected using the 515/30 and 610LP emission filters for YFP and chlorophyll autofluorescence, respectively.

For Arabidopsis transformations, cotyledons were taken for analysis 3 days after infiltration. Confocal microscopy observations were performed using Zeiss LSM700 microscope equipped with 20x and 40x EC Plan-Neofluar objectives. YFP and chlorophyll fluorescence was excited using 488 $\mathrm{nm}$ laser. Signals were detected using 507-561BP (YFP) and 652-682BP (chlorophyll) filters with beam splitter set at $601 \mathrm{~nm}$.

\section{Thermotolerance tests}

Arabidopsis seeds of WT, cia2-2, cil-1, and cia2-2 cil-1 plants were surface sterilized by soaking in a $50 \%(\mathrm{v} / \mathrm{v})$ commercial bleach solution for $7 \mathrm{~min}$. The seeds were then rinsed five times with sterile water and resuspended in $1 \mathrm{ml}$ of $0.1 \%$ (w/v) agarose solution. The seeds were stratified for 2 days at $4{ }^{\circ} \mathrm{C}$. They were then placed evenly onto plates with $35 \mathrm{ml}$ of $1 / 2 \mathrm{MS}$ medium with agar at $\mathrm{pH}$ 5.8. Each plate was divided into four equal parts, one part per genotype, and approximately 25 
682 seeds of every genotype were sown on a single plate. The plates were parafilm sealed to prevent

683 dehydration of growing plants. The seeds were germinated and cultivated for 7 days under standard 684 growth conditions.

After 7 days of in vitro cultivation, the seedlings were subjected to thermotolerance assay according to a previously described procedure (Silva-Correia et al., 2014). Heat treatment was performed under ambient laboratory light after $3 \mathrm{~h}$ of light photoperiod. Parafilm-sealed plates with grown seedlings were set in a water bath preheated to $45^{\circ} \mathrm{C}$ for 20,30 , or $40 \mathrm{~min}$. After the treatment, the plates were transferred again to the growth chamber where the plants were cultivated for subsequent 7 days.

The survival rate of seedlings of individual genotypes was evaluated from the heat-treated plants' ability to produce new green leaves 7 days after the treatment. The plants were imaged, and

693 the proportion of seedlings survived after heat treatment and all germinated plants was calculated for 694 each genotype at every timepoint.

\section{Accession numbers}

The following gene names are used in Figure 7: Rps6 (AT1G64510), Rpl11 (AT1G32990), Rpl18 (AT1G48350), Rpl28 (AT2G33450), Rpl3 (AT2G43030), Rpl21 (AT1G35680), PSRP2 (AT3G52150), Rpl9 (AT3G44890), Rps17 (AT1G79850), Rps5 (AT2G33800), Rps10 (AT3G13120), Rpl17 (AT3G54210), Rpl13 (AT1G78630), PSRP4 (AT2G38140), Rps9 (AT1G74970), Rps21 (AT3G27160), rpl2 (ATCG01310 and ATCG00830), Rps20 (AT3G15190), Rpl19.1 (AT4G17560), Rpl19.2 (AT5G47190), Rpl29 (AT5G65220), rpl32 (ATCG01020), DG1 (AT5G67570), PPR2 (AT3G06430), SVR7 (AT4G16390), PPR5 (AT4G39620), EMB2279 (AT1G30610), PDM1/SEL1 (AT4G18520) PPR596 (AT1G80270), HCF152 (AT3G09650), PSRP1 (AT5G24490), PBR1 (AT1G71720), IF3-4 (AT4G30690), IF3-2 (AT2G24060), cpHSC70-2 (AT5G49910), and SVR3 (AT5G13650).

\section{Acknowledgments}

We thank Prof. Ralph Bock for psrp5-R1 and rps 17-1 seeds. We thank Dr. Peter Robert Kindgren for help with $P P R$ gene analysis and Dr. Anna Kozłowska-Makulska for technical help during the cHL screening. P.G., P.B., J.M., M.G., M.Z., and S.K. acknowledge financial support from the Polish National Science Center (Narodowe Centrum Nauki; OPUS6, UMO2013/11/B/NZ3/00973, MAESTRO6 UMO-2014/14/A/NZ1/00218 given to S.K. and SONATA14 UMO-2018/31/D/NZ3/03296 given to M.G.). L.B.S. acknowledges financial support from the Independent Research Fund Denmark (Danmarks Frie Forskningsfond; 7014-00322B). C.W. acknowledges financial support from the Academy of Finland (Decision 294580), D.L. is grateful for support from the German Science Foundation (DFG, grant TR175 C05). 


\section{Short legends for Supporting Information}

Table S1. List of genes selected from targeted reverse genetic screen for subcellular localization experiments.

Table S2. List of primers used in this study.

Figure S1. Examples of results obtained from the initial reverse genetic screening in UV-AB and high-light experiments.

Figure S2. Isolation and characterization of cia2-2 cil-2 double mutant.

Figure S3. High-light (HL) and UV-AB susceptibility of cia2-2 cil-2.

Figure S4. CIA2 and CIL are required for optimal photosynthesis in Arabidopsis.

Figure S5. Analysis of rRNA and NPQ in plastid translation mutants.

Data S1. List of T-DNA mutants used in targeted reverse genetic screen.

Data S2. List of genes differentially expressed in cia2-2 cil-1 compared to Col-0.

\section{Conflict of interest}

No conflicts of interest.

\section{Author contributions}

P.G., C.W., and S.K. formulated the hypothesis and conceived the research plan. P.G., P.B., L.B.S., M.Z., M.G. and J.M. performed the experiments; P.G., P.B., and L.B.S. analyzed the data; P.G., C.W., D.L. and S.K. supervised the analysis; and P.G., P.B., C.W., L.B.S., and S.K. wrote the article, with contributions from all authors.

\section{References}

Barajas-López, J. de D., Blanco, N.E. and Strand, Å. (2013) Plastid-to-nucleus communication, signals controlling the running of the plant cell. Biochim. Biophys. Acta, 1833, 425-437.

Barkan, A. and Small, I. (2014) Pentatricopeptide repeat proteins in plants. Annu. Rev. Plant Biol., 65, 415-442.

Bernacki, M.J., Czarnocka, W., Szechyńska-Hebda, M., Mittler, R. and Karpiński, S. (2019) Biotechnological potential of LSD1, EDS1, and PAD4 in the improvement of crops and industrial plants. Plants, 8, 290.

Białasek, M., Górecka, M., Mittler, R. and Karpiński, S. (2017) Evidence for the involvement of electrical, calcium and ROS signaling in the systemic regulation of non-photochemical quenching and photosynthesis. Plant Cell Physiol., 58, 207-215. 
Bieri, P., Leibundgut, M., Saurer, M., Boehringer, D. and Ban, N. (2017) The complete structure of the chloroplast $70 \mathrm{~S}$ ribosome in complex with translation factor pY. EMBO J., 36, 475-486.

Bollenbach, T.J., Lange, H., Gutierrez, R., Erhardt, M., Stern, D.B. and Gagliardi, D. (2005) RNR1, a 3'-5' exoribonuclease belonging to the RNR superfamily, catalyzes 3' maturation of chloroplast ribosomal RNAs in Arabidopsis thaliana. Nucleic Acids Res., 33, 2751-2763.

Bollenbach, T.J., Schuster, G., Portnoy, V. and Stern, D.B. (2007) Processing, degradation, and polyadenylation of chloroplast transcripts. Topics in Current Genetics. pp. 175-211.

Börner T. (2017) The discovery of plastid-to-nucleus retrograde signaling - a personal perspective. Protoplasma, 254, 1845-1855.

Bradbeer, J.W., Atkinson, Y.E., Borner, T., and Hagemann, R. (1979). Cytoplasmic synthesis of plastid polypeptides may be controlled by plastid-synthesised RNA. Nature 279, 816-817.

Burdiak, P., Rusaczonek, A., Witoń, D., Głów, D. and Karpiński, S. (2015) Cysteine-rich receptor-like kinase CRK5 as a regulator of growth, development, and ultraviolet radiation responses in Arabidopsis thaliana. J. Exp. Bot., 66, 3325-3337.

Caldwell, C.R. (1993) Ultraviolet-induced photodegradation of cucumber (Cucumis sativus L.) microsomal and soluble protein tryptophanyl residues in vitro. Plant Physiol., 101, 947-953.

Caplan, J.L., Kumar, A.S., Park, E., Padmanabhan, M.S., Hoban, K., Modla, S., Czymmek, K. and Dinesh-Kumar, S.P. (2015) Chloroplast stromules function during innate immunity. Dev. Cell, 34, 45-57.

Casati, P. and Walbot, V. (2004) Crosslinking of ribosomal proteins to RNA in maize ribosomes by UV-B and its effects on translation. Plant Physiol., 136, 3319-3332.

Chan, K.X., Mabbitt, P.D., Phua, S.Y., et al. (2016) Sensing and signaling of oxidative stress in chloroplasts by inactivation of the SAL1 phosphoadenosine phosphatase. Proc. Natl Acad. Sci. USA, 113, E4567-E4576.

Chi, W., Sun, X. and Zhang, L. (2013) Intracellular signaling from plastid to nucleus. Annu. Rev. Plant Biol., 64, 559-582.

Chotewutmontri, P. and Barkan, A. (2018) Multilevel effects of light on ribosome dynamics in chloroplasts program genome-wide and psbA-specific changes in translation. PLOS Genet., 14, e1007555.

Clercq, I. De, Vermeirssen, V., Aken, O. Van, et al. (2013) The membrane-bound NAC transcription factor ANAC013 functions in mitochondrial retrograde regulation of the oxidative stress response in Arabidopsis. Plant Cell, 25, 3472-3490.

Daniell, H., Lin, C.-S., Yu, M. and Chang, W.-J. (2016) Chloroplast genomes: diversity, evolution, and applications in genetic engineering. Genome Biol., 17, 134.

Dickinson, P.J., Kumar, M., Martinho, C., et al. (2018) Chloroplast signaling gates thermotolerance in Arabidopsis. Cell Rep., 22, 1657-1665. 
Distelbarth, H., Nägele, T. and Heyer, A.G. (2013) Responses of antioxidant enzymes to cold and high light are not correlated to freezing tolerance in natural accessions of Arabidopsis thaliana. Plant Biol., 15, 982-990.

Dogra, V., Li, M., Singh, S., Li, M. and Kim, C. (2019) Oxidative post-translational modification of EXECUTER1 is required for singlet oxygen sensing in plastids. Nat. Commun., 10, 2834.

Eberhard, S., Drapier, D. and Wollman, F.A. (2002) Searching limiting steps in the expression of chloroplast-encoded proteins: Relations between gene copy number, transcription, transcript abundance and translation rate in the chloroplast of Chlamydomonas reinhardtii. Plant J., 31, 149-160.

Estavillo, G.M., Chan, K.X., Phua, S.Y. and Pogson, B.J. (2013) Reconsidering the nature and mode of action of metabolite retrograde signals from the chloroplast. Front. Plant Sci., 3, 1-9.

Estavillo, G.M., Crisp, P. a., Pornsiriwong, W., et al. (2011) Evidence for a SAL1-PAP chloroplast retrograde pathway that functions in drought and high light signaling in Arabidopsis. Plant Cell, 23, 3992-4012.

Exposito-Rodriguez, M., Laissue, P.P., Yvon-Durocher, G., Smirnoff, N. and Mullineaux, P.M. (2017) Photosynthesis-dependent $\mathrm{H}_{2} \mathrm{O}_{2}$ transfer from chloroplasts to nuclei provides a high-light signalling mechanism. Nat. Commun., 8, 49.

Ferreyra, M.L.F., Pezza, A., Biarc, J., Burlingame, A.L. and Casati, P. (2010) Plant L10 ribosomal proteins have different roles during development and translation under ultraviolet-B stress. Plant Physiol., 153, 1878-1894.

Finkelstein, R., Lynch, T., Reeves, W., Petitfils, M. and Mostachetti, M. (2011) Accumulation of the transcription factor ABA-insensitive (ABI) 4 is tightly regulated post-transcriptionally. $J$. Exp. Bot., 62, 3971-3979.

Foyer, C.H., Karpinska, B. and Krupinska, K. (2014) The functions of WHIRLY1 and REDOXRESPONSIVE TRANSCRIPTION FACTOR 1 in cross tolerance responses in plants: a hypothesis. Philos Trans R Soc Lond B Biol Sci 369, 20130226.

Fristedt, R., Scharff, L.B., Clarke, C. a, Wang, Q., Lin, C., Merchant, S.S. and Bock, R. (2014) $\mathrm{RBF} 1$, a plant homolog of the bacterial ribosome-binding factor RbfA, acts in processing of the chloroplast 16S ribosomal RNA. Plant Physiol., 164, 201-15.

Gawronski, P., Witon, D., Vashutina, K., Bederska, M., Betlinski, B., Rusaczonek, A. and Karpinski, S. (2014) Mitogen-activated protein kinase 4 is a salicylic acid-independent regulator of growth but not of photosynthesis in Arabidopsis. Mol. Plant, 7, 1151-1166.

Gilroy, S., Białasek, M., Suzuki, N., Górecka, M., Devireddy, A.R., Karpiński, S. and Mittler, R. (2016) ROS, calcium, and electric signals: Key mediators of rapid systemic signaling in plants. Plant Physiol., 171, 1606-1615.

Grabowski, E., Miao, Y., Mulisch, M. and Krupinska, K. (2008) Single-stranded DNA-binding protein Whirly1 in barley leaves is located in plastids and the nucleus of the same cell. Plant Physiol., 147, 1800-1804. 
Guo, H., Feng, P., Chi, W., et al. (2016) Plastid-nucleus communication involves calciummodulated MAPK signalling. Nat. Commun., 7, 1-15.

Herdean, A., Teardo, E., Nilsson, A.K., et al. (2016) A voltage-dependent chloride channel finetunes photosynthesis in plants. Nat. Commun., 7, 11654.

Hess, W.R., Müller, A., Nagy, F., and Börner, T. (1994). Ribosome- deficient plastids affect transcription of light induced nuclear genes: Genetic evidence for a plastid derived signal. Mol. Gen. Genet. 242, 505-512

Hollósy, F. (2002) Effects of ultraviolet radiation on plant cells. Micron, 33, 179-197.

Huang, D., Lan, W., Li, D., Deng, B., Lin, W., Ren, Y. and Miao, Y. (2018) WHIRLY1 occupancy affects histone lysine modification and WRKY53 transcription in Arabidopsis developmental manner. Front Plant Sci. 19, 1503.

Jarvis, P. (2008) Targeting of nucleus-encoded proteins to chloroplasts in plants. New Phytol., 179, $257-285$.

Jarvis, P. and Robinson, C. (2004) Mechanisms of protein import and routing in chloroplasts. Curr. Biol., 14, 1064-1077.

Jaspers, P., Blomster, T., Brosché, M., et al. (2009) Unequally redundant RCD1 and SRO1 mediate stress and developmental responses and interact with transcription factors. Plant J., 60, 268-279.

Jin, J., Zhang, H., Kong, L., Gao, G. and Luo, J. (2014) PlantTFDB 3.0: A portal for the functional and evolutionary study of plant transcription factors. Nucleic Acids Res., 42, 11821187.

Kacprzak, S.M., Mochizuki, N., Naranjo, B., Xu, D., Leister, D., Kleine, T., Okamoto, H. and Terry, M.J. (2019) Plastid-to-nucleus retrograde signalling during chloroplast biogenesis does not require ABI4. Plant Physiol., 179, 18-23.

Karpiński, S., Escobar, C., Karpinska, B., Creissen, G. and Mullineaux, P.M. (1997) Photosynthetic electron transport regulates the expression of cytosolic ascorbate peroxidase genes in Arabidopsis during excess light stress. Plant Cell, 9, 627-640.

Karpiński, S., Reynolds, H., Karpinska, B., Wingsle, G., Creissen, G. and Mullineaux, P.M. (1999) Systemic signaling and acclimation in response to excess excitation energy in Arabidopsis. Science, 284, 654-657.

Klinkenberg, J. (2014). Extraction of chloroplast proteins from transiently transformed Nicotiana benthamiana leaves. Bio-protocol, 4, e1238.

Klinkenberg, J., Faist, H., Saupe, S., Lambertz, S., Krischke, M., Stingl, N., Fekete, A., Mueller, M. J., Feussner, I., Hedrich, R. and Deeken, R. (2014). Two fatty acid desaturases, STEAROYL-ACYL CARRIER PROTEIN Delta9-DESATURASE6 and FATTY ACID DESATURASE3, are involved in drought and hypoxia stress signaling in Arabidopsis crown galls. Plant Physiol., 164, 570-583. 
Krause, K., Oetke, S. and Krupinska, K. (2012) Dual targeting and retrograde translocation: Regulators of plant nuclear gene expression can be sequestered by plastids. Int. J. Mol. Sci., 13, 11085-11101.

Krupinska, K., Oetke, S., Desel, C., Mulisch, M., Schäfer, A., Hollmann, J., Kumlehn, J. and Hensel G. (2014) WHIRLY1 is a major organizer of chloroplast nucleoids. Front Plant Sci., 5, 432.

Kulasek, M., Bernacki, M.J., Ciszak, K., Witoń, D. and Karpiński, S. (2016) Contribution of PsbS function and stomatal conductance to foliar temperature in higher plants. Plant Cell Physiol., 57, 1495-1509.

Lee, K.P., Kim, C., Landgraf, F. and Apel, K. (2007) EXECUTER1- and EXECUTER2dependent transfer of stress-related signals from the plastid to the nucleus of Arabidopsis thaliana. Proc. Natl Acad. Sci. USA, 104, 10270-10275.

Li, J.F., Park, E., Arnim, A.G. Von and Nebenführ, A. (2009a) The FAST technique: A simplified Agrobacterium-based transformation method for transient gene expression analysis in seedlings of Arabidopsis and other plant species. Plant Methods, 5, 1-15.

Li, M., Hensel, G., Mascher, M., et al. (2019) Leaf variegation and impaired chloroplast development caused by a truncated CCT domain gene in albostrians Barley. Plant Cell, 31, 1430-1445.

Li, Z., Wakao, S., Fischer, B.B. and Niyogi, K.K. (2009b) Sensing and responding to excess light. Annu. Rev. Plant Biol., 60, 239-260.

Liere, K. and Börner, T. (2007) Transcription and transcriptional regulation in plastids. In Systems Biology. pp. 121-174.

Love, M.I., Huber, W. and Anders, S. (2014) Moderated estimation of fold change and dispersion for RNA-seq data with DESeq2. Genome Biol., 15, 550.

Maréchal, A Parent, J-S., Véronneau-Lafortune, F., Joyeux, A., Lang, F. and Brisson, N. (2009) Whirly proteins maintain plastid genome stability in Arabidopsis. Proc. Natl Acad. Sci. USA, 106, 14693-14698.

Mateo, A., Mühlenbock, P., Rustérucci, C., Chang, C.C., Miszalski, Z., Karpinska, B., Parker, J.E., Mullineaux, P.M. and Karpinski, S. (2004) LESION SIMULATING DISEASE 1 is required for acclimation to conditions that promote excess excitation energy. Plant Physiol., 136, 2818-2830.

Miao, Y., Jiang, J., Ren, Y. and Zhao, Z. (2013) The single-stranded DNA-binding protein WHIRLY1 represses WRKY53 expression and delays leaf senescence in a developmental stage-dependent manner in Arabidopsis. Plant Physiol., 163, 746-756.

Miyata, K., Ikeda, H., Nakaji, M., Kanel, D.R. and Terashima, I. (2015) Rate constants of PSII photoinhibition and its repair, and PSII fluorescence parameters in field plants in relation to their growth light environments. Plant Cell Physiol., 56, 1841-1854. 
Mühlenbock, P., Szechynska-Hebda, M., Plaszczyca, M., Baudo, M., Mullineaux, P.M., Parker, J.E., Karpinska, B. and Karpiński, S. (2008) Chloroplast signaling and LESION SIMULATING DISEASE1 regulate crosstalk between light acclimation and immunity in Arabidopsis. Plant Cell, 20, 2339-2356.

Mullineaux, P.M., Karpiński, S. and Baker, N.R. (2006) Spatial dependence for hydrogen peroxide-directed signaling in light-stressed plants. Plant Physiol., 141, 346-350.

Myouga, F., Akiyama, K., Tomonaga, Y., Kato, A., Sato, Y., Kobayashi, M., Nagata, N., Sakurai, T. and Shinozaki, K. (2013) The chloroplast function database II: A comprehensive collection of homozygous mutants and their phenotypic/genotypic traits for nuclear-encoded chloroplast proteins. Plant Cell Physiol., 54, 1-10.

Nakagawa, T., Kurose, T., Hino, T., et al. (2007) Development of series of gateway binary vectors, pGWBs, for realizing efficient construction of fusion genes for plant transformation. $J$. Biosci. Bioeng., 104, 34-41.

Nakai, M. (2015) The TIC complex uncovered: The alternative view on the molecular mechanism of protein translocation across the inner envelope membrane of chloroplasts. Biochim. Biophys. Acta, 1847, 957-967.

Ng, S., Ivanova, A., Duncan, O., et al. (2013) A membrane-bound NAC transcription factor, ANAC017, mediates mitochondrial retrograde signaling in Arabidopsis. Plant Cell, 25, 3450 3471.

Nickelsen, J., Bohne, A.-V. and Westhoff, P. (2014) Chloroplast gene expression-translation. In Plastid Biology. New York, NY: Springer New York, pp. 49-78.

Nishimura, K., Ashida, H., Ogawa, T. and Yokota, A. (2010) A DEAD box protein is required for formation of a hidden break in Arabidopsis chloroplast 23S rRNA. Plant J., 63, 766-777.

Niyogi, K.K., Li, X.P., Rosenberg, V. and Jung, H.S. (2005) Is PsbS the site of nonphotochemical quenching in photosynthesis? J. Exp. Bot., 56, 375-382.

Nørholm, M.H. (2010) A mutant Pfu DNA polymerase designed for advanced uracil-excision DNA engineering. BMC Biotechnol., 10, 21.

Nour-Eldin, H.H., Hansen, B.G., Nørholm, M.H.H., Jensen, J.K. and Halkier, B.A. (2006) Advancing uracil-excision based cloning towards an ideal technique for cloning PCR fragments. Nucleic Acids Res., 34, e122-e122.

Page, M.T., Kacprzak, S.M., Mochizuki, N., Okamoto, H., Smith, A.G. and Terry, M.J. (2017) Seedlings lacking the PTM protein do not show a genomes uncoupled (gun) mutant phenotype. Plant Physiol., 174, 21-26.

Paila, Y.D., Richardson, L.G.L. and Schnell, D.J. (2015) New insights into the mechanism of chloroplast protein import and its integration with protein quality control, organelle biogenesis and development. J. Mol. Biol., 427, 1038-1060.

Parker, N., Wang, Y. and Meinke, D. (2014) Natural variation in sensitivity to a loss of chloroplast translation in Arabidopsis. Plant Physiol., 166, 2013-2027. 
Patro, R., Duggal, G., Love, M.I., Irizarry, R.A. and Kingsford, C. (2017) Salmon provides fast and bias-aware quantification of transcript expression. Nat. Methods, 14, 417-419.

Pérez-Rodríguez, P., Riaño-Pachón, D.M., Corrêa, L.G.G., Rensing, S.A., Kersten, B. and Mueller-Roeber, B. (2009) PlnTFDB: Updated content and new features of the plant transcription factor database. Nucleic Acids Res., 38, 822-827.

Pesaresi, P., Masiero, S., Eubel, H., Braun, H.-P., Bhushan, S., Glaser, E., Salamini, F. and Leister, D. (2006) Nuclear photosynthetic gene expression is synergistically modulated by rates of protein synthesis in chloroplasts and mitochondria. Plant Cell, 18, 970-991.

Pesaresi, P., Varotto, C., Meurer, J., Jahns, P., Salamini, F. and Leister, D. (2001) Knock-out of the plastid ribosomal protein L11 in Arabidopsis: Effects on mRNA translation and photosynthesis. Plant J., 27, 179-189.

Prikryl, J., Watkins, K. P., Friso, G., Wijk, K. J. and Barkan A. (2008) A member of the Whirly family is a multifunctional RNA- and DNA-binding protein that is essential for chloroplast biogenesis. Nucleic Acids Res., 36, 5152-5165.

Pogson, B.J., Woo, N.S., Förster, B. and Small, I.D. (2008) Plastid signalling to the nucleus and beyond. Trends Plant Sci., 13, 602-9.

Ramakers, C., Ruijter, J.M., Deprez, R.H.L. and Moorman, A.F. (2003) Assumption-free analysis of quantitative real-time polymerase chain reaction (PCR) data. Neurosci. Lett., 339, $62-66$.

Ramel, F., Birtic, S., Ginies, C., Soubigou-Taconnat, L., Triantaphylides, C. and Havaux, M. (2012) Carotenoid oxidation products are stress signals that mediate gene responses to singlet oxygen in plants. Proc. Natl Acad. Sci. USA, 109, 5535-5540.

Romani, I., Tadini, L., Rossi, F., Masiero, S., Pribil, M., Jahns, P., Kater, M., Leister, D. and Pesaresi, P. (2012) Versatile roles of Arabidopsis plastid ribosomal proteins in plant growth and development. Plant J., 72, 922-934.

Ruckle, M.E., Burgoon, L.D., Lawrence, L.A., Sinkler, C.A. and Larkin, R.M. (2012) Plastids are major regulators of light signaling in Arabidopsis. Plant Physiol., 159, 366-390.

Scharff, L.B. and Bock, R. (2014) Synthetic biology in plastids. Plant J., 78, 783-798.

Schreiber, U. and Klughammer, C. (2008) New accessory for the DUAL-PAM-100: The P515/535 module and examples of its application. PAM Appl. Notes, 1, 1-10.

Schwacke, R., Schneider, A., Graaff, E. Van Der, Fischer, K., Catoni, E., Desimone, M., Frommer, W.B., Flügge, U.I. and Kunze, R. (2003) ARAMEMNON, a novel database for Arabidopsis integral membrane proteins. Plant Physiol., 131, 16-26.

Schwacke, R., Fischer, K., Ketelsen, B., Krupinska, K., Krause, K. (2007) Comparative survey of plastid and mitochondrial targeting properties of transcription factors in Arabidopsis and rice. Mol. Genet. Genomics., 277:631-646. 
Shapiguzov, A., Vainonen, J.P., Hunter, K., et al. (2019) Arabidopsis RCD1 coordinates chloroplast and mitochondrial functions through interaction with ANAC transcription factors. Elife, 8, 1-35.

Silva-Correia, J., Freitas, S., Tavares, R.M., Lino-Neto, T. and Azevedo, H. (2014) Phenotypic analysis of the Arabidopsis heat stress response during germination and early seedling development. Plant Methods, $10,7$.

Silvestro, D., Andersen, T.G., Schaller, H. and Jensen, P.E. (2013) Plant sterol metabolism. $\Delta 7$ sterol-C5-desaturase (STE1/DWARF7), $\Delta 5,7$-sterol- $\Delta 7$-reductase (DWARF5) and $\Delta 24$-sterol$\triangle 24$-reductase (DIMINUTO/DWARF1) show multiple subcellular localizations in Arabidopsis thaliana (Heynh). PLoS One, 8, e56429.

Smirnoff, N. and Arnaud, D. (2019) Hydrogen peroxide metabolism and functions in plants. New Phytol., 221, 1197-1214.

Soneson, C., Love, M.I. and Robinson, M.D. (2016) Differential analyses for RNA-seq: transcript-level estimates improve gene-level inferences. F1000Research, 4, 1521.

Souza, A. de, Wang, J.-Z. and Dehesh, K. (2017) Retrograde signals: integrators of interorganellar communication and orchestrators of plant development. Annu. Rev. Plant Biol., 68, 85-108.

Strayer, C., Oyama, T., Schultz, T., Raman, R., Somers, D., Mas, P., Panda, S., Kreps, J. and Kay, S. (2000) Cloning of the Arabidopsis clock gene TOC1, an autoregulatory response regulator homolog. Science, 289, 768-771.

Sugiura, M. (2014) Plastid mRNA translation. In Chloroplast Biotechnology. pp. 73-91.

Sumanta, N., Haque, C., Nishika, J. and Suprakash, R. (2014) Spectrophotometric analysis of chlorophylls and carotenoids from commonly grown fern species by using various extracting solvents. Res. J. Chem. Sci., 4, 2231-606.

Sun, C.-W., Chen, L.-J., Lin, L.-C. and Li, H. (2001) Leaf-specific upregulation of chloroplast translocon genes by a CCT motif-containing protein, CIA2. Plant Cell, 13, 2053-2061.

Sun, C.-W., Huang, Y.-C. and Chang, H.-Y. (2009a) CIA2 coordinately up-regulates protein import and synthesis in leaf chloroplasts. Plant Physiol., 150, 879-888.

Sun, Q., Zybailov, B., Majeran, W., Friso, G., Olinares, P.D.B. and Wijk, K.J. van (2009b) PPDB, the Plant Proteomics Database at Cornell. Nucleic Acids Res., 37, D969-D974.

Sun, X., Feng, P., Xu, X., Guo, H., Ma, J., Chi, W., Lin, R., Lu, C. and Zhang, L. (2011) A chloroplast envelope-bound PHD transcription factor mediates chloroplast signals to the nucleus. Nat. Commun., 2, 477.

Tadini, L., Pesaresi, P., Kleine, T., et al. (2016) GUN1 controls accumulation of the plastid ribosomal protein $\mathrm{S} 1$ at the protein level and interacts with proteins involved in plastid protein homeostasis. Plant Physiol., 170, 1817-30. 
1005

1006

1007

1008

1009

1010

1011

1012

1013

1014

1015

1016

1017

1018

1019

1020

1021

1022

1023

1024

1025

1026

1027

1028

1029

1030

1031

1032

1033

1034

1035

1036

1037

1038

1039

1040

1041

1042

Te, J., Melcher, U., Howard , A. and Verchot-Lubicz J. (2005) Soilborne wheat mosaic virus (SBWMV) 19K protein belongs to a class of cysteine rich proteins that suppress RNA silencing. Virol. J., 2, 18.

Tiller, N., Weingartner, M., Thiele, W., Maximova, E., Schottler, M.A. and Bock, R. (2012) The plastid-specific ribosomal proteins of Arabidopsis thaliana can be divided into nonessential proteins and genuine ribosomal proteins. Plant J., 69, 302-316.

Tokumaru, M., Adachi, F., Toda, M., et al. (2017) Ubiquitin-proteasome dependent regulation of the GOLDEN2-LIKE 1 transcription factor in response to plastid signals. Plant Physiol., 173, $524-535$.

Wagner, R. and Pfannschmidt, T. (2006) Eukaryotic transcription factors in plastids Bioinformatic assessment and implications for the evolution of gene expression machineries in plants. Gene, 381, 62-70.

Wang, L., Kim, C., Xu, X., Piskurewicz, U., Dogra, V. and Singh, S. (2016) Singlet oxygen- and EXECUTER1-mediated signaling is initiated in grana margins and depends on the. Proc. Natl Acad. Sci. USA, 113, E3792-E3800.

Waters, M.T., Wang, P., Korkaric, M., Capper, R.G., Saunders, N.J. and Langdale, J. a (2009) GLK transcription factors coordinate expression of the photosynthetic apparatus in Arabidopsis. Plant Cell, 21, 1109-1128.

Wituszyńska, W., Ślesak, I., Vanderauwera, S., et al. (2013) LESION SIMULATING DISEASE1, ENHANCED DISEASE SUSCEPTIBILITY1, and PHYTOALEXIN DEFICIENT4 conditionally regulate cellular signaling homeostasis, photosynthesis, water use efficiency, and seed yield in Arabidopsis. Plant Physiol., 161, 1795-1805.

Wituszyńska, W., Szechyńska-Hebda, M., Sobczak, M., Rusaczonek, A., KozlowskaMakulska, A., Witoń, D. and Karpiński, S. (2015) LESION SIMULATING DISEASE 1 And ENHANCED DISEASE SUSCEPTIBILITY 1 differentially regulate UV-C-induced photooxidative stress signalling and programmed cell death in Arabidopsis thaliana. Plant, Cell Environ., 38, 315-330.

Wu, G.-Z., Chalvin, C., Hoelscher, M.P., Meyer, E.H., Wu, X.N. and Bock, R. (2018) Control of retrograde signaling by rapid turnover of GENOMES UNCOUPLED 1. Plant Physiol., 176, $2472-2495$.

Wu, G., Meyer, E.H., Richter, A.S., et al. (2019) Control of retrograde signalling by protein import and cytosolic folding stress. Nat. Plants, 5, 525-538.

Yabuta, Y., Motoki, T., Yoshimura, K., Takeda, T., Ishikawa, T. and Shigeoka, S. (2002) Thylakoid membrane-bound ascorbate peroxidase is a limiting factor of antioxidative systems under photo-oxidative stress. Plant J., 32, 915-925.

Yasumura, Y., Moylan, E.C. and Langdale, J.A. (2005) A conserved transcription factor mediates nuclear control of organelle biogenesis in anciently diverged land plants. Plant Cell, 17, 1894-1907. 
1043 Yu Q-B., Li G., Wang G., et al. (2008) Construction of a chloroplast protein interaction network 1044 and functional mining of photosynthetic proteins in Arabidopsis thaliana. Cell Res., 18, 1007 $1045 \quad 1019$

1046 Zoschke, R. and Bock, R. (2018) Chloroplast translation: structural and functional organization, 1047 operational control and regulation. Plant Cell, 30, 745-770. 


\section{Figure legends}

1050 Figure 1. Sub-cellular localization of CIA2 in Arabidopsis and tobacco. CIA2:YFP (a, c, d) and 1051 CIA2 ${ }^{1-100}:$ YFP $(\mathbf{b}, \mathbf{e})$ fusion proteins were transiently expressed in Arabidopsis seedlings $(\mathbf{a}, \mathbf{b})$ and 1052 in Nicotiana benthamiana leaves (c-e) under control of the $35 \mathrm{~S}$ constitutive promoter. d presents 1053 chloroplast localized CIA2:YFP at larger magnification. Pictures were taken 3 days post infiltration 1054 using confocal microscopy. Magenta and green colors represent chlorophyll and YFP fluorescence, 1055 respectively. White scale bars indicate $10 \mu \mathrm{m}$. (f) Localization of CIA2 ${ }^{1-100}$ :YFP in stroma 1056 (p35S::CIA2 ${ }^{1-100}:$ YFP was expressed in Col-0). Total protein extracts, chloroplast stroma and 1057 thylakoid membranes (TM) were isolated from Col- 0 and plants expressing CIA2 ${ }^{1-100}:$ YFP under the 1058 control of $35 \mathrm{~S}$ promoter. Protein extracts were separated by SDS-PAGE and analyzed on Western 1059 blots using antibodies against GFP, large subunit of RuBisCo (RbcL) and D1 protein of PSII.

1060 Figure 2. Isolation and characterization of cia2-2 cil-1 double mutant. (a, b) Isolation of cia2-4 1061 mutant in reverse genetic screening using UV-AB (a) and high light in combination with low temperature (cHL) (b). (c) In the upper part, a schematic representation of CIA2 and CIL genes is presented. Black and white rectangles represent exons and untranslated regions, respectively. The lower part shows the analysis of functional domains in CIA2 and CIL proteins: cTP-chloroplast transit peptide (functional - dark green, predicted - light green), NLS-nuclear localization signal, CCT-putative active domain in CIA2 and CIL. The CCT domain contains NLS; however, it is not shown in the diagram for simplicity. (d) Phenotypes of 4-week-old plants grown in long-day conditions. cia2-2 and cia2-2 cil-1 plants were paler than the rest of analyzed genotypes; thus, the content of photosynthetic pigments was measured and shown in (e). (f) Expression of CIA2 and CIL in the analyzed genotypes was measured using qRT-PCR with gene-specific primers. Locations of 1071 primers are depicted in panel $(\mathbf{c})$. (g, h) Complementation of cia2-2 phenotype with ectopic 1072 expression of CIA2 under $35 \mathrm{~S}$ and native promoter. (g) Relative expression of CIA2 in analyzed lines. (h) Relative content of photosynthetic pigments in complementation lines. In (a), (e) and (f) statistical significance (ANOVA and Tukey HSD test) is shown relative to Col-0 $(* * p<0.01 ; * * * p<0.001$ ). In (g) and (h) statistical significance (ANOVA and Tukey HSD test) is shown relative to cia2-2 (**p $<0.01 ; * * * p<0.001)$ and to Col-0 $\left({ }^{\wedge} p<0.05 ;{ }^{\wedge \wedge} p<0.01 ;{ }^{\wedge \wedge \wedge} p<0.001\right)$.

1077 Figure 3. Sub-cellular localization of CIL in Arabidopsis and tobacco. CIL:YFP (a, b) and CIL ${ }^{1-}$ 1078 ${ }^{100}:$ YFP (c) fusion proteins were transiently expressed in Arabidopsis seedlings (a, c) and in Nicotiana benthamiana leaves (b) under control of the 35S constitutive promoter. Pictures were taken 3 days post infiltration using confocal microscopy. Magenta and green colors represent chlorophyll and YFP fluorescence, respectively. White scale bars indicate $10 \mu \mathrm{m}$. 
1082 Figure 4. CIA2 and CIL are required for resistance to UV-AB. Mature plants were exposed to UV$1083 \mathrm{AB}$, and the plants' performance was assessed using chlorophyll $a$ fluorescence (a and b) and ion 1084 leakage (c). (a) Maximum efficiency of PSII $\left(F_{\mathrm{v}} / F_{\mathrm{m}}\right)$, nonregulated energy dissipation (Y(NO)), and nonphotochemical quenching (NPQ) were measured before and after UV-AB treatment (1 and 2 days). Each point represents mean \pm SEM of at least eight plants. (b) $F_{\mathrm{v}} / F_{\mathrm{m}}$ of control (UV-) and UV-

1087 AB-treated (UV +) plants of Col-0 and cia2-2 cil-1 plants. Values of $F_{\mathrm{v}} / F_{\mathrm{m}}$ are shown in pseudocolor 1088 scale. (c) Ion leakage of control (UV-, $n=5)$ and UV-AB-treated (UV,$+ n=18$ ) plants is shown as 1089 a percentage of total ion leakage. Statistical significance (ANOVA and Tukey HSD test) is shown 1090 relative to Col-0 $(* p<0.05 ; * * * p<0.001)$ and to control conditions $\left({ }^{\wedge \wedge \wedge} p<0.001\right)$.

1091 Figure 5. High-light (HL) susceptibility of cia2-2 cil-1. (a) Representation of Arabidopsis rosette 1092 with the young leaves marked with blue, dashed line. $(\mathbf{b}, \mathbf{c}, \mathbf{d})$ Maximum efficiency of PSII $\left(F_{\mathrm{v}} / F_{\mathrm{m}}\right)$ 1093 measured in plants (b) exposed to blue HL $\left(1100 \mu \mathrm{mol} \mathrm{m}^{-2} \mathrm{~s}^{-1}\right)$ for a specified time $(\mathrm{n}=4-8$ plants $)$, 1094 (c) during recovery after HL stress, $\mathrm{n}=6-8,(\mathbf{d})$ and treated with inhibitors influencing the production 1095 of ROS in chloroplasts $(n=12-22)$. In each plot, points represent mean \pm SEM measured in 1096 independent plants (b and c) or leaf disks (d). Statistical significance (ANOVA and Tukey HSD test) 1097 is shown relative to Col- $0(* p<0.05 ; * * p<0.01 ; * * p<0.001)$.

1098 Figure 6. CIA2 and CIL are required for optimal photosynthesis in Arabidopsis. (a) 1099 Nonphotochemical quenching (NPQ) in analyzed genotypes. Points represent mean \pm SEM of the 1100 whole rosette and only young leaves. (b) NPQ of Col-0 and cia2-2 cil-1. (c) Analysis of 1101 electrochromic pigment shift (ECS, P515) at 160 and $660 \mu \mathrm{mol} \mathrm{m}^{-2} \mathrm{~s}^{-1}$ of actinic light. For simplicity, 1102 only Col-0 and cia2-2 cil-1 are shown. ST-single turnover flash. Total ECS $\left(\mathrm{ECS}_{\mathrm{t}}\right)(\mathbf{d})$ and $\Delta \mathrm{pH}(\mathbf{e})$ 1103 in analyzed genotypes at 160 and $660 \mu \mathrm{mol} \mathrm{m}^{-2} \mathrm{~s}^{-1}$. Box plots represent values of 10 independent 1104 plants. (f) $\mathrm{CO}_{2}$ assimilation as a function of light intensity. (g) $\mathrm{CO}_{2}$ assimilation as a function of $\mathrm{CO}_{2}$ 1105 concentration. In (f) and (g), values represent mean \pm SEM of 7-9 plants. Statistical significance 1106 (ANOVA and Tukey HSD test) is shown relative to Col-0 $(* p<0.05 ; * * p<0.01 ; * * p<0.001$ ).

1107 Figure 7. Characterization of chloroplast translation in analyzed genotypes. (a) Expression analysis 1108 of genes encoding chloroplast ribosomal proteins. Levels of analyzed transcripts were determined 1109 using qRT-PCR and normalized to two house-keeping genes (PP2AA3 and TIP4IL). Bars indicate 1110 mean values \pm SD (three independent biological replicates). (b) Expression profile (RNA-seq) of genes 1111 involved in the regulation of plastid translation. (c) Polysome analysis reveals slower translation of $1112 p s b D$ mRNA in cia2-2 cil-1 double mutant as compared to Col-0. Gray triangles indicate the density 1113 of sucrose gradient. Methylene blue-stained 18S rRNA is shown as a loading control. (d) 1114 Susceptibility to chloroplast translation inhibitor (spectinomycin, $1.25 \mathrm{mg} / \mathrm{L}$ ) of analyzed genotypes. 
1115 statistical significance (ANOVA and Tukey HSD test) is shown relative to Col-0 $\left(* p<0.05\right.$; $*^{*} p<$ $11160.01 ; * * * p<0.001)$.

1117 Figure 8. Maturation of plastid rRNAs. (a) Plastid rRNA operon. Mature forms of 16 and 23S rRNA 1118 are shown. Precursor of $23 \mathrm{~S}$ rRNA is processed and cleaved into three parts: $0.4,1.1$, and $1.3 \mathrm{~kb}$. (b) 1119 Relative abundance of precursor 23S and 16S rRNAs was measured using capillary electrophoresis 1120 and normalized to the cytoplasmic 18S rRNA. Values represent mean values \pm SEM $(n=3)$. (c) 1121 Maturation and abundance of plastid rRNA were measured using Northern blot indicating that the $11222.4 \mathrm{~kb}$ form of 23S rRNA is accumulated in cia2-2 cil-1. (d) Determination of relative amounts of 1123 23S rRNA forms with qRT-PCR and primers (depicted in panel (a)) specific to each of cleaved 1124 fragments (primers A, B, and C) and flanking "hidden breaks" (primers AB and BC). Values were 1125 normalized to the level of $16 \mathrm{~S}$ rRNA and represent mean values $\pm \mathrm{SD}(\mathrm{n}=3)$. Statistical significance 1126 (ANOVA and Tukey HSD test) is shown relative to Col-0 $(* p<0.05$; ** $p<0.01$; *** $p<0.001$ ).

1127 Figure 9. CIA2 and CIL negatively regulate tolerance to heat shock. (a) Gene ontology (GO) analysis 1128 of genes significantly induced in cia2-2 cil-1 compared to Col-0 in the RNA-seq experiment. Ten 1129 most significantly overrepresented GO terms are shown. The number of genes in each group is shown 1130 in color of the bar. (b) Validation of expression of heat shock marker genes using qRT-PCR in 1131 analyzed genotypes and additional allele (cia2-3) in Ler-0 background $(n=3)$. Error bars represent $1132 \pm \mathrm{SD}$. (c and d) Thermo tolerance was tested in seedlings grown in vitro. Plants were exposed to 45 $1133{ }^{\circ} \mathrm{C}$ for a specific period of time. (c) Pictures of plants grown in control conditions 7 days after heat 1134 shock. (d) Survival rate of control and heat shock-treated plants. Error bars represent \pm SEM (n $\geq 3$ 1135 plates) from two independent experiments (in total at least 75 seedlings were analyzed per genotype 1136 and treatment). Statistical significance (ANOVA and Tukey HSD test) is shown relative to 1137 corresponding WT $(* * * p<0.001)$. 


\section{Figures}

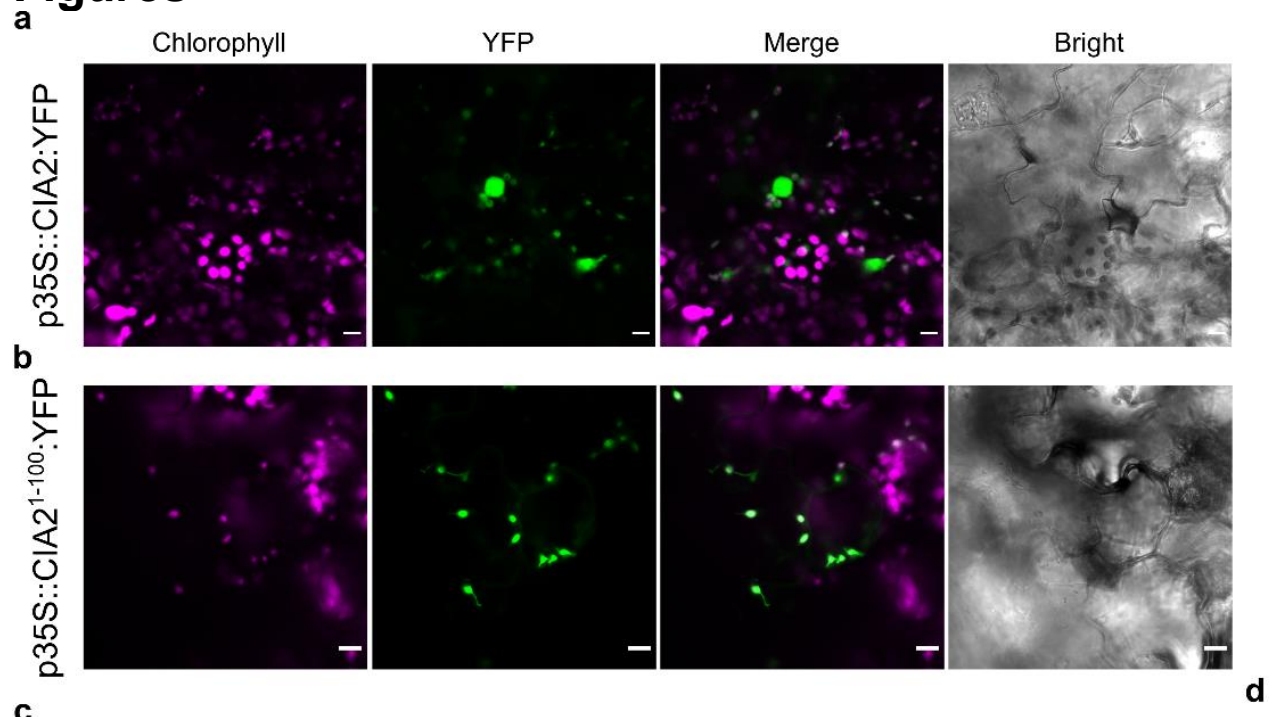

c

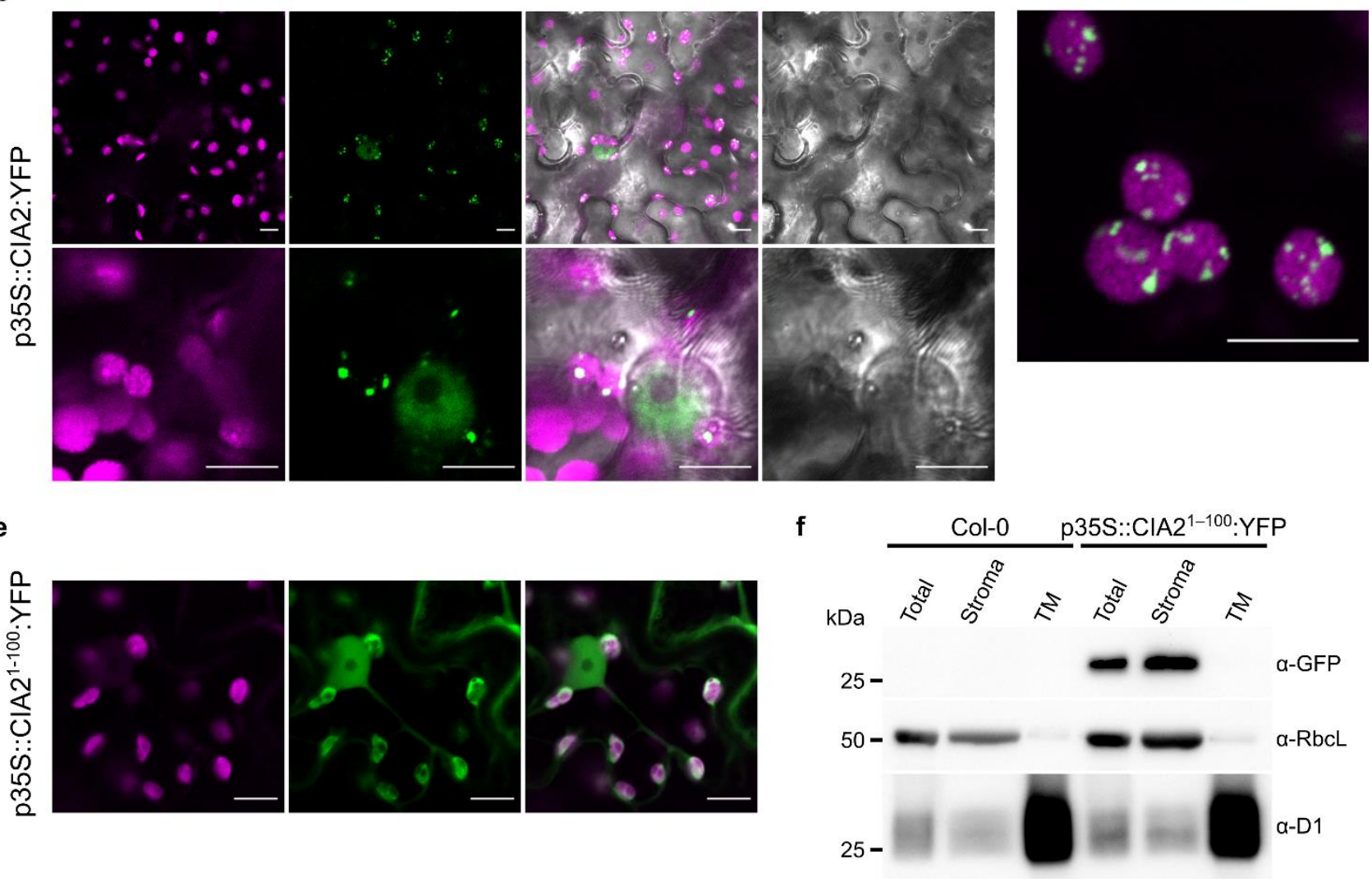

1140 Figure 1. Sub-cellular localization of CIA2 in Arabidopsis and tobacco. CIA2:YFP (a, c, d) and 1141 CIA2 ${ }^{1-100}:$ YFP $(\mathbf{b}, \mathbf{e})$ fusion proteins were transiently expressed in Arabidopsis seedlings $(\mathbf{a}, \mathbf{b})$ and 1142 in Nicotiana benthamiana leaves (c-e) under control of the $35 \mathrm{~S}$ constitutive promoter. d presents 1143 chloroplast localized CIA2:YFP at larger magnification. Pictures were taken 3 days post infiltration 1144 using confocal microscopy. Magenta and green colors represent chlorophyll and YFP fluorescence, 1145 respectively. White scale bars indicate $10 \mu \mathrm{m}$. (f) Localization of CIA2 ${ }^{1-100}$ :YFP in stroma 1146 (p35S::CIA2 ${ }^{1-100}:$ YFP was expressed in Col-0). Total protein extracts, chloroplast stroma and 1147 thylakoid membranes (TM) were isolated from Col-0 and plants expressing CIA2 ${ }^{1-100}:$ YFP under the 
1148 control of 35 S promoter. Protein extracts were separated by SDS-PAGE and analyzed on Western 1149 blots using antibodies against GFP, large subunit of RuBisCo (RbcL) and D1 protein of PSII. 1150 

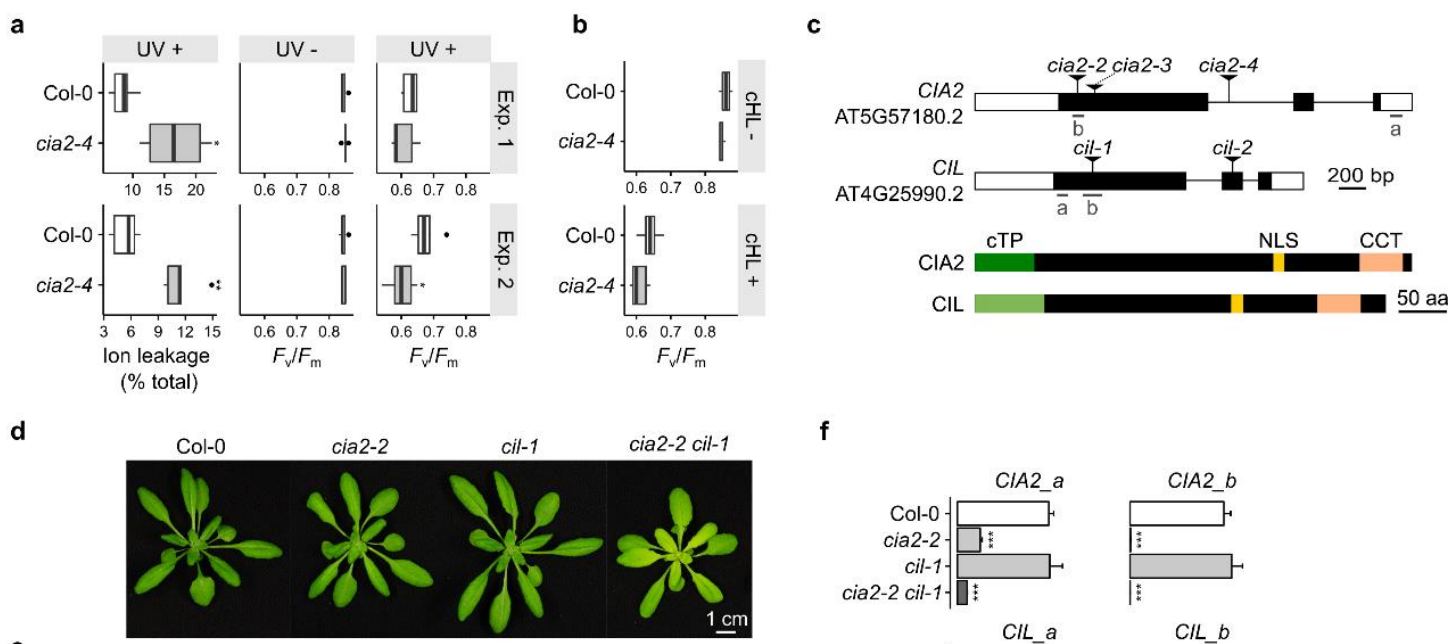

$\mathbf{f}$
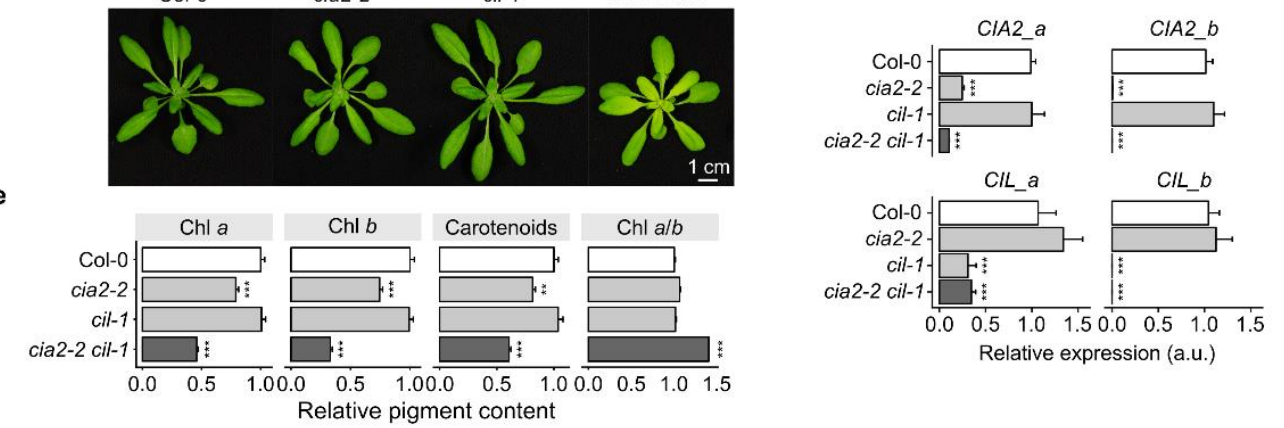

g

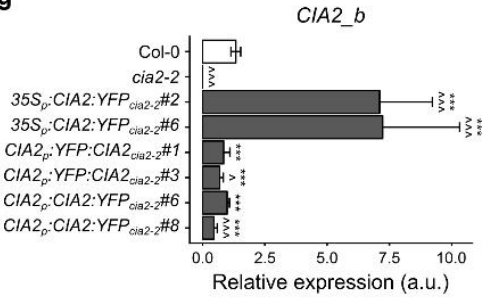

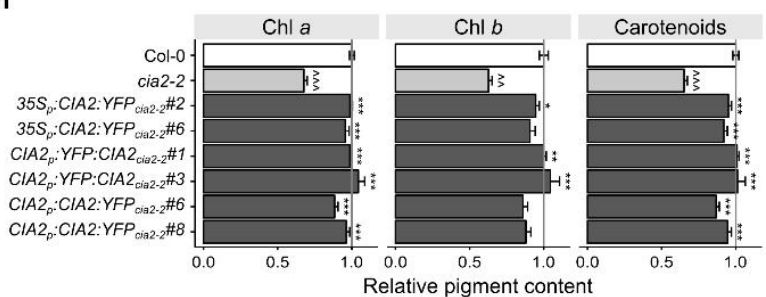

1152 Figure 2. Isolation and characterization of cia2-2 cil-1 double mutant. (a, b) Isolation of cia2-4

1153 mutant in reverse genetic screening using UV-AB (a) and high light in combination with low

1154 temperature (cHL) (b). (c) In the upper part, a schematic representation of CIA2 and CIL genes is

1155 presented. Black and white rectangles represent exons and untranslated regions, respectively. The

1156 lower part shows the analysis of functional domains in CIA2 and CIL proteins: cTP-chloroplast

1157 transit peptide (functional - dark green, predicted - light green), NLS-nuclear localization signal,

1158 CCT-putative active domain in CIA2 and CIL. The CCT domain contains NLS; however, it is not

1159 shown in the diagram for simplicity. (d) Phenotypes of 4-week-old plants grown in long-day

1160 conditions. cia2-2 and cia2-2 cil-1 plants were paler than the rest of analyzed genotypes; thus, the

1161 content of photosynthetic pigments was measured and shown in (e). (f) Expression of CIA2 and CIL

1162 in the analyzed genotypes was measured using qRT-PCR with gene-specific primers. Locations of

1163 primers are depicted in panel $(\mathbf{c})$. (g, h) Complementation of cia2-2 phenotype with ectopic

1164 expression of CIA2 under 35S and native promoter. (g) Relative expression of CIA2 in analyzed lines.

1165 (h) Relative content of photosynthetic pigments in complementation lines. In (a), (e) and (f) statistical

1166 significance (ANOVA and Tukey HSD test) is shown relative to Col-0 (** $p<0.01$; *** $p<0.001)$. 
1167 In (g) and (h) statistical significance (ANOVA and Tukey HSD test) is shown relative to cia2-2 $(* * p$ $\left.1168<0.01 ; *^{* *} p<0.001\right)$ and to Col-0 $\left({ }^{\wedge} p<0.05 ;{ }^{\wedge} p<<0.01 ;{ }^{\wedge \wedge \wedge} p<0.001\right)$. 

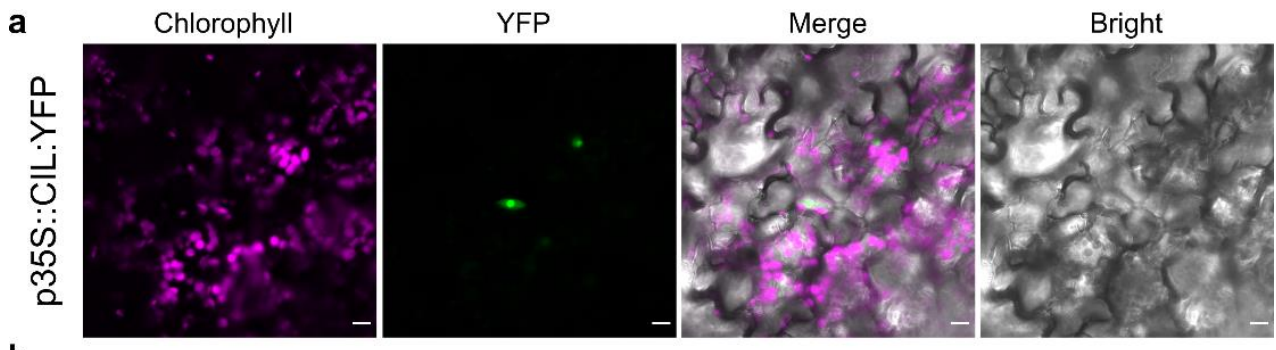

b

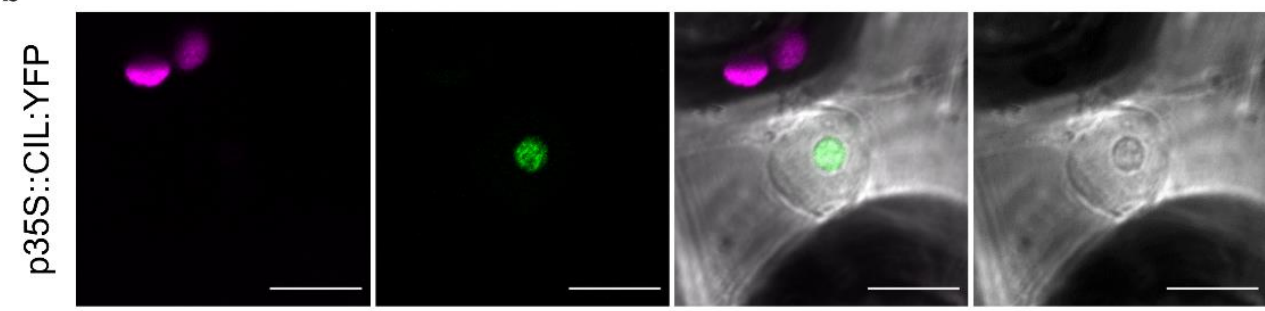

C
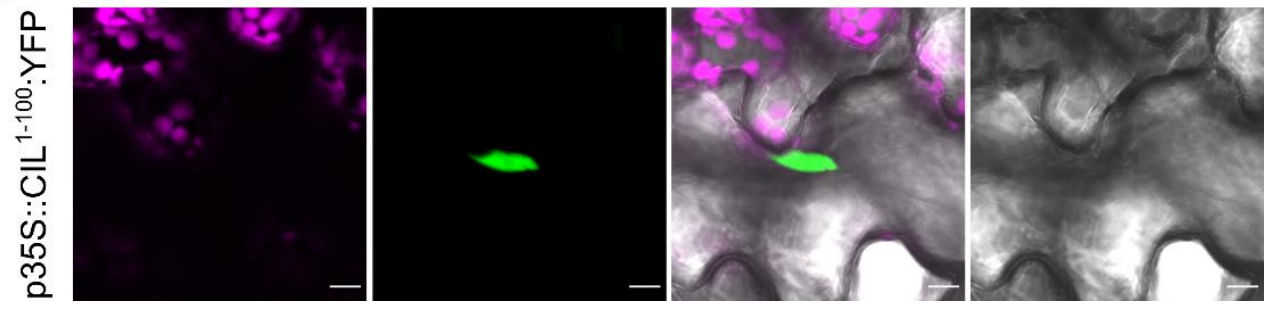

1171 Figure 3. Sub-cellular localization of CIL in Arabidopsis and tobacco. CIL:YFP (a, b) and CIL ${ }^{1-}$ $1172{ }^{100}$ :YFP (c) fusion proteins were transiently expressed in Arabidopsis seedlings (a, c) and in Nicotiana 1173 benthamiana leaves (b) under control of the 35S constitutive promoter. Pictures were taken 3 days 1174 post infiltration using confocal microscopy. Magenta and green colors represent chlorophyll and YFP 1175 fluorescence, respectively. White scale bars indicate $10 \mu \mathrm{m}$. 

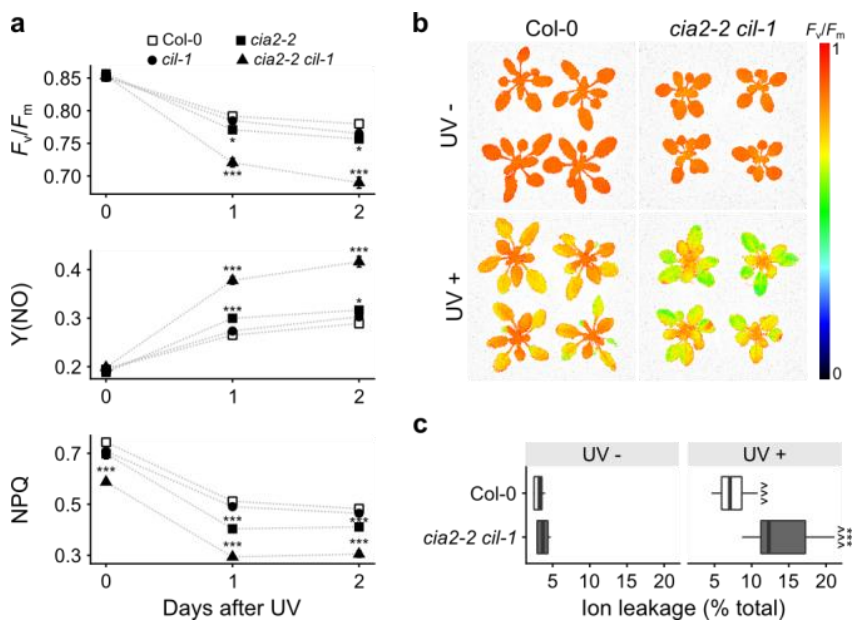

1177 Figure 4. CIA2 and CIL are required for resistance to UV-AB. Mature plants were exposed to UV$1178 \mathrm{AB}$, and the plants' performance was assessed using chlorophyll $a$ fluorescence (a and $\mathbf{b}$ ) and ion 1179 leakage (c). (a) Maximum efficiency of PSII $\left(F_{\mathrm{v}} / F_{\mathrm{m}}\right)$, nonregulated energy dissipation (Y(NO)), and 1180 nonphotochemical quenching (NPQ) were measured before and after UV-AB treatment (1 and 2 1181 days). Each point represents mean \pm SEM of at least eight plants. (b) $F_{\mathrm{v}} / F_{\mathrm{m}}$ of control (UV-) and UV1182 AB-treated (UV +) plants of Col-0 and cia2-2 cil-1 plants. Values of $F_{\mathrm{v}} / F_{\mathrm{m}}$ are shown in pseudocolor 1183 scale. (c) Ion leakage of control (UV-, $n=5$ ) and UV-AB-treated (UV,$+ n=18$ ) plants is shown as 1184 a percentage of total ion leakage. Statistical significance (ANOVA and Tukey HSD test) is shown 1185 relative to Col- $0(* p<0.05 ; * * * p<0.001)$ and to control conditions $\left({ }^{\wedge \wedge \wedge} p<0.001\right)$. 
a

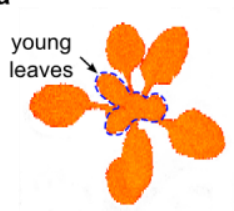

c

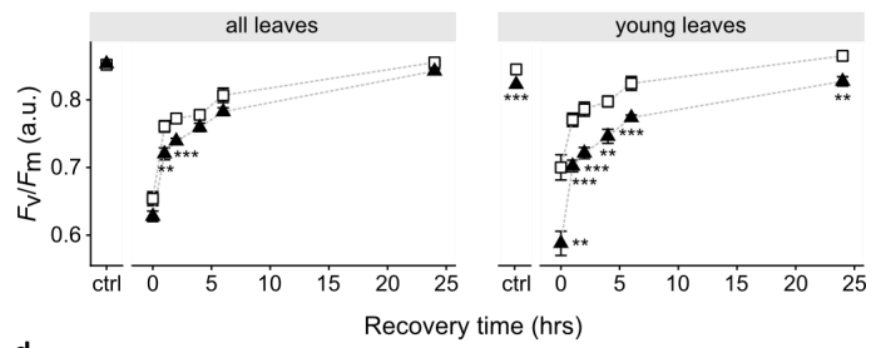

d

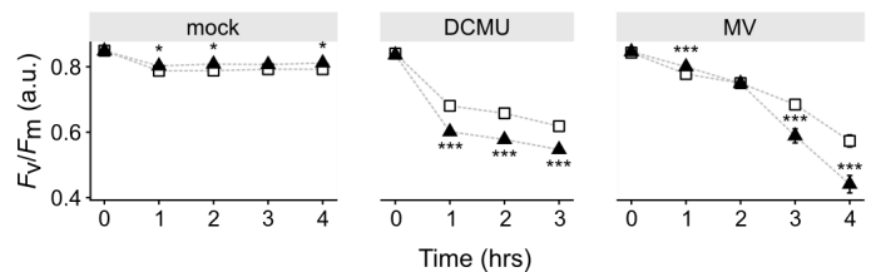

b $\quad$ Col-0 $\Delta$ cia2-2 cil-1

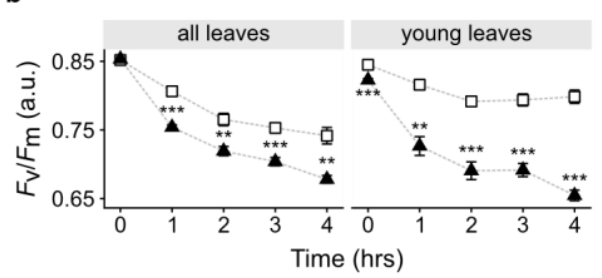

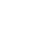



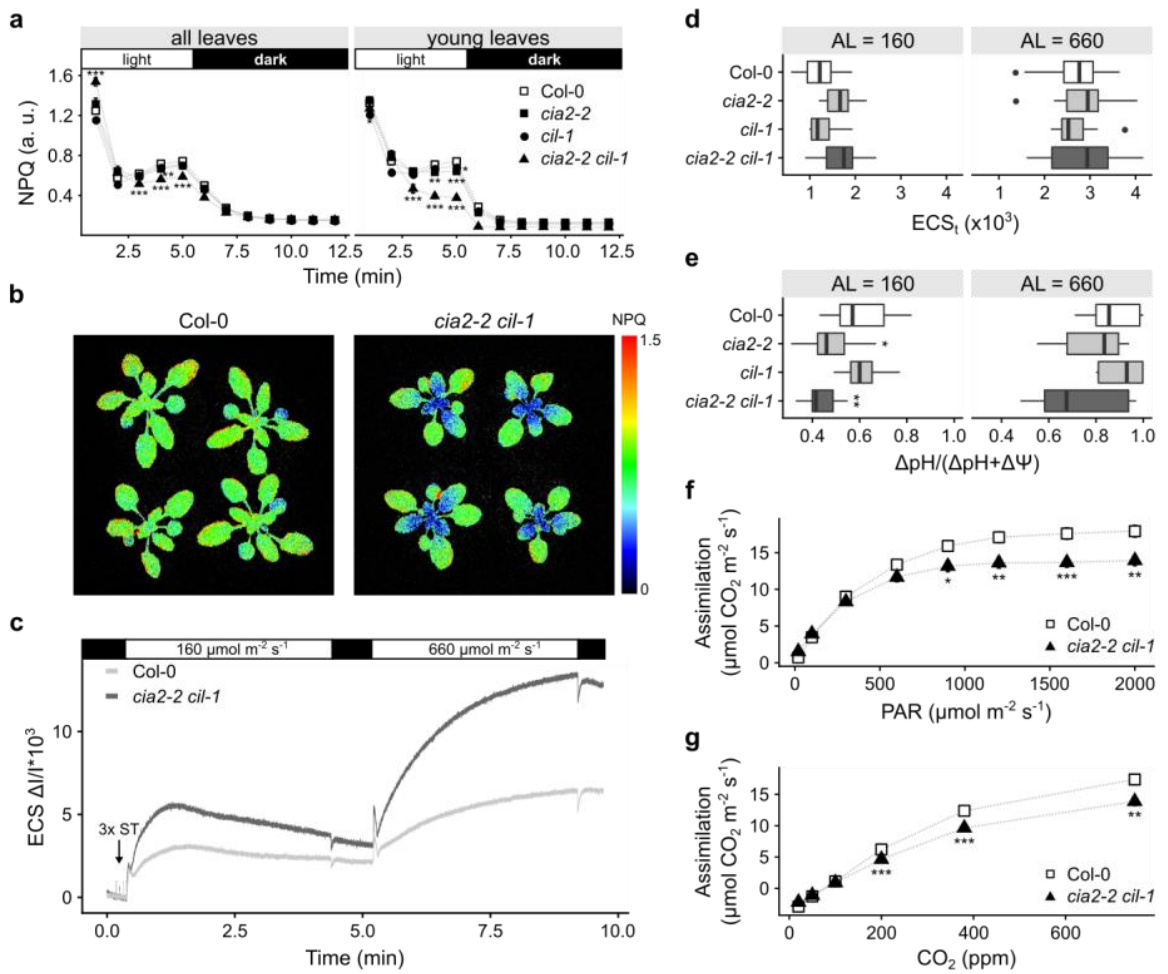

1195 Figure 6. CIA2 and CIL are required for optimal photosynthesis in Arabidopsis. (a) 1196 Nonphotochemical quenching (NPQ) in analyzed genotypes. Points represent mean \pm SEM of the 1197 whole rosette and only young leaves. (b) NPQ of Col-0 and cia2-2 cil-1. (c) Analysis of 1198 electrochromic pigment shift (ECS, P515) at 160 and $660 \mu \mathrm{mol} \mathrm{m}^{-2} \mathrm{~s}^{-1}$ of actinic light. For simplicity, 1199 only Col-0 and cia2-2 cil-1 are shown. ST-single turnover flash. Total ECS $\left(\mathrm{ECS}_{\mathrm{t}}\right)(\mathbf{d})$ and $\Delta \mathrm{pH}(\mathbf{e})$ 1200 in analyzed genotypes at 160 and $660 \mu \mathrm{mol} \mathrm{m} \mathrm{m}^{-2} \mathrm{~s}^{-1}$. Box plots represent values of 10 independent 1201 plants. (f) $\mathrm{CO}_{2}$ assimilation as a function of light intensity. (g) $\mathrm{CO}_{2}$ assimilation as a function of $\mathrm{CO}_{2}$ 1202 concentration. In (f) and (g), values represent mean \pm SEM of 7-9 plants. Statistical significance 1203 (ANOVA and Tukey HSD test) is shown relative to Col-0 $(* p<0.05 ; * * p<0.01 ; * * * p<0.001)$. 
a
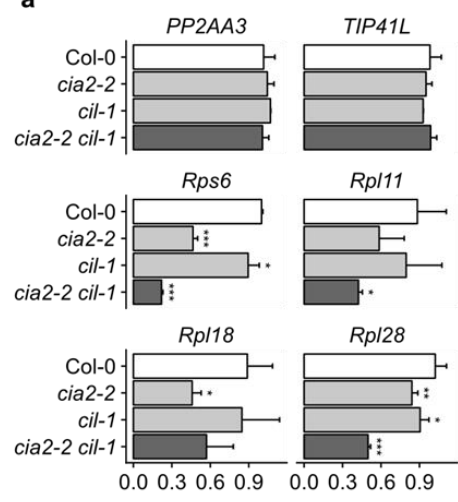

Relative expression (a.u.)

c

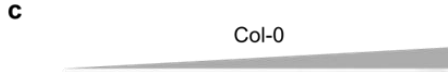

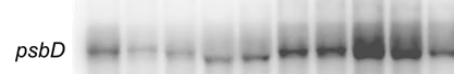

$18 \mathrm{~S}$ b
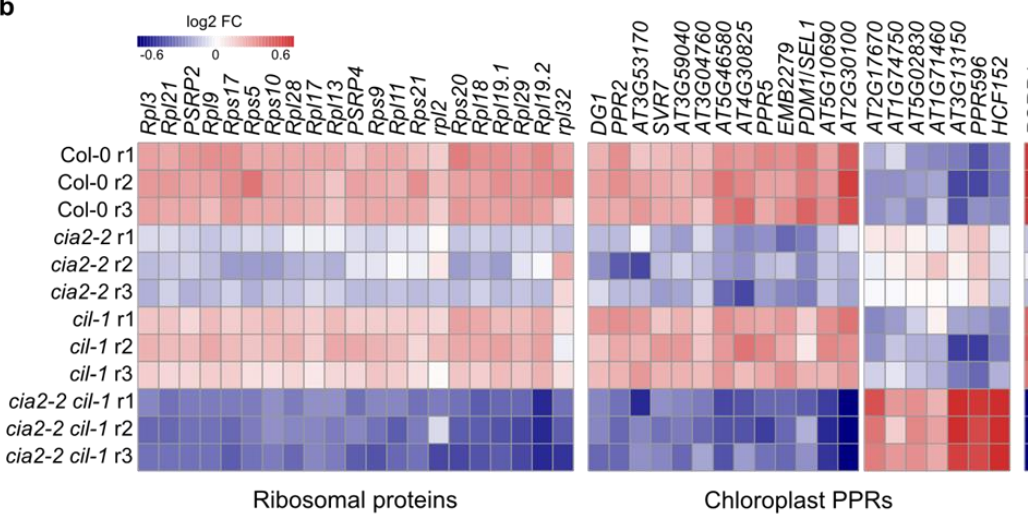

Chloroplast PPRs

cia2-2 cil-1

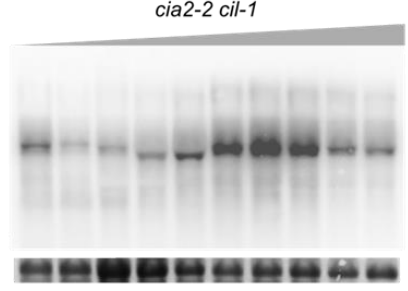

d

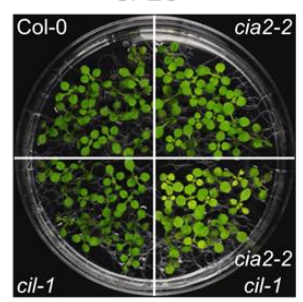

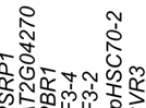

<造는 엉

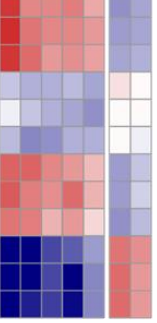

Other

1205 Figure 7. Characterization of chloroplast translation in analyzed genotypes. (a) Expression analysis

1206 of genes encoding chloroplast ribosomal proteins. Levels of analyzed transcripts were determined 1207 using qRT-PCR and normalized to two house-keeping genes (PP2AA3 and TIP41L). Bars indicate 1208 mean values \pm SD (three independent biological replicates). (b) Expression profile (RNA-seq) of genes 1209 involved in the regulation of plastid translation. (c) Polysome analysis reveals slower translation of 1210 psbD mRNA in cia2-2 cil-1 double mutant as compared to Col-0. Gray triangles indicate the density 1211 of sucrose gradient. Methylene blue-stained 18S rRNA is shown as a loading control. (d) 1212 Susceptibility to chloroplast translation inhibitor (spectinomycin, $1.25 \mathrm{mg} / \mathrm{L}$ ) of analyzed genotypes. 1213 statistical significance (ANOVA and Tukey HSD test) is shown relative to Col-0 $(* p<0.05 ; * *<$ $12140.01 ; * * * p<0.001)$. 


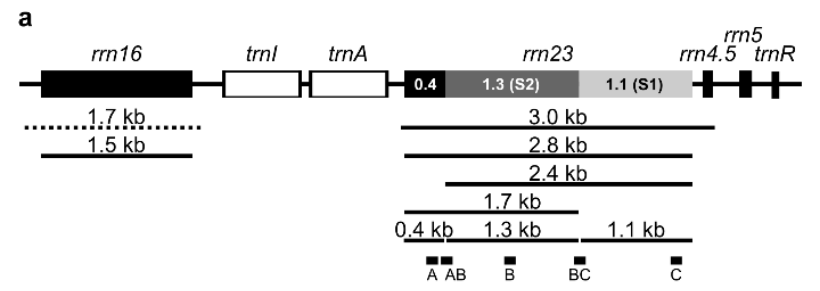

b

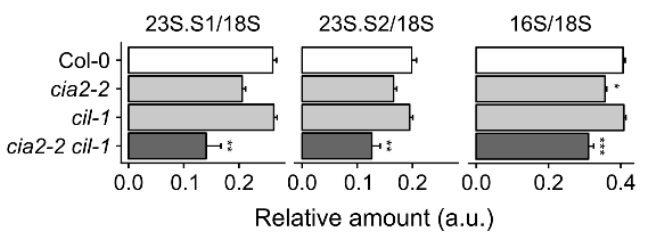

d

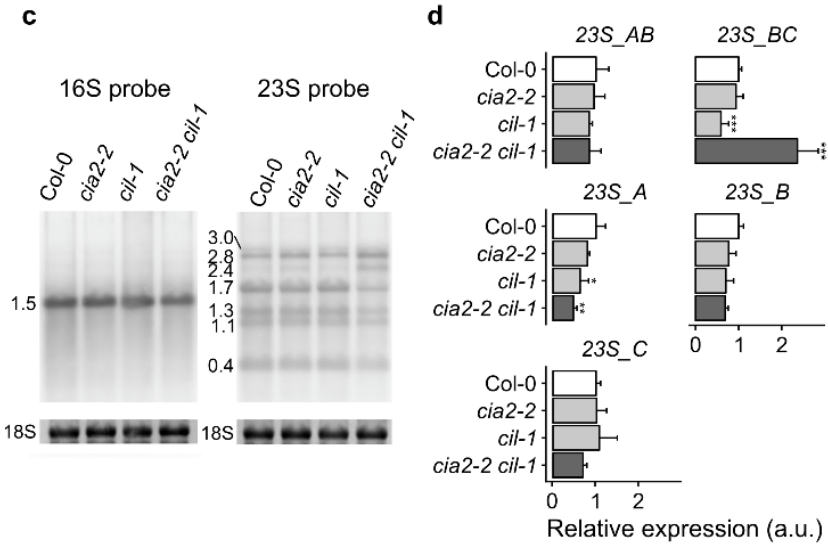

1216 Figure 8. Maturation of plastid rRNAs. (a) Plastid rRNA operon. Mature forms of 16 and 23S rRNA 1217 are shown. Precursor of $23 \mathrm{~S}$ rRNA is processed and cleaved into three parts: $0.4,1.1$, and $1.3 \mathrm{~kb}$. (b) 1218 Relative abundance of precursor 23S and 16S rRNAs was measured using capillary electrophoresis 1219 and normalized to the cytoplasmic 18S rRNA. Values represent mean values \pm SEM $(\mathrm{n}=3)$. $(\mathbf{c})$ 1220 Maturation and abundance of plastid rRNA were measured using Northern blot indicating that the $12212.4 \mathrm{~kb}$ form of $23 \mathrm{~S}$ rRNA is accumulated in cia2-2 cil-1. (d) Determination of relative amounts of 1222 23S rRNA forms with qRT-PCR and primers (depicted in panel (a)) specific to each of cleaved 1223 fragments (primers A, B, and C) and flanking "hidden breaks" (primers AB and BC). Values were 1224 normalized to the level of $16 \mathrm{~S}$ rRNA and represent mean values \pm SD $(n=3)$. Statistical significance 1225 (ANOVA and Tukey HSD test) is shown relative to Col- $0(* p<0.05 ; * * p<0.01 ; * * * p<0.001)$. 
a

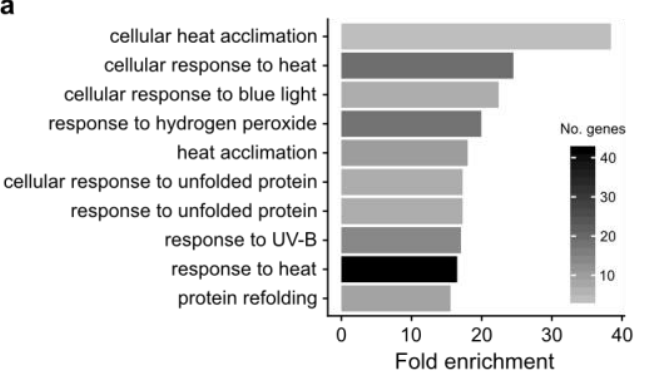

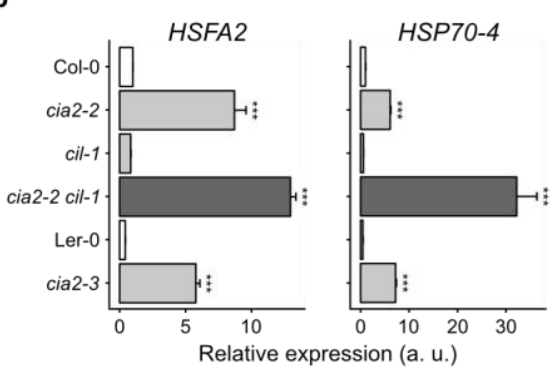

c
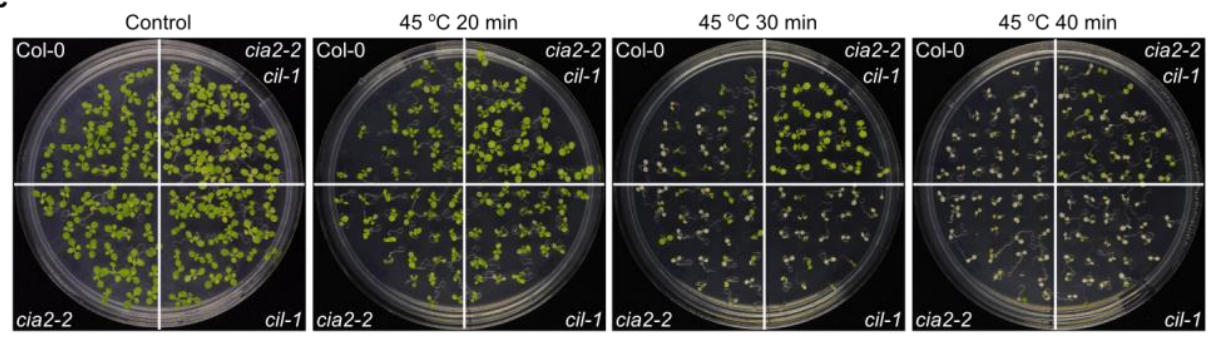

d
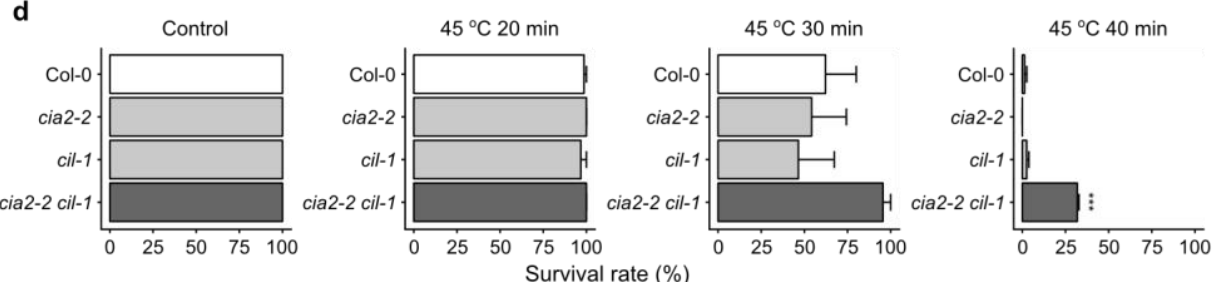

1227 Figure 9. CIA2 and CIL negatively regulate tolerance to heat shock. (a) Gene ontology (GO) analysis 1228 of genes significantly induced in cia2-2 cil-1 compared to Col-0 in the RNA-seq experiment. Ten 1229 most significantly overrepresented GO terms are shown. The number of genes in each group is shown 1230 in color of the bar. (b) Validation of expression of heat shock marker genes using qRT-PCR in 1231 analyzed genotypes and additional allele (cia2-3) in Ler-0 background $(n=3)$. Error bars represent $1232 \pm$ SD. (c and d) Thermo tolerance was tested in seedlings grown in vitro. Plants were exposed to 45 $1233{ }^{\circ} \mathrm{C}$ for a specific period of time. (c) Pictures of plants grown in control conditions 7 days after heat 1234 shock. (d) Survival rate of control and heat shock-treated plants. Error bars represent \pm SEM ( $\mathrm{n} \geq 3$ 1235 plates) from two independent experiments (in total at least 75 seedlings were analyzed per genotype 1236 and treatment). Statistical significance (ANOVA and Tukey HSD test) is shown relative to 1237 corresponding WT $(* * * p<0.001)$. 